\title{
Synthesis of Biaryls and Polyaryls by Ligand-Free Suzuki Reaction in Aqueous Phase
}

\author{
Leifang Liu, Yuhong Zhang*, Bingwei Xin
}

Department of Chemistry, Zhejiang University, Hangzhou 310027, P.R. China.

E-mail:yhzhang@zjuem.zju.edu.cn

\section{Supporting Information}

\section{Content}

General procedure for Suzuki reaction................................. 2

Characterization data of the product of the Suzuki reaction................S3

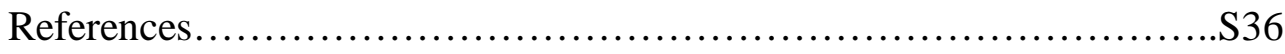


General Starting materials and solvents were purchased from common commercial sources and were used without additional purification. The gas chromatography analysis was performed on a GC instrument. ${ }^{1} \mathrm{H}$ NMR spectra were recorded at $500 \mathrm{MHz}$ or $400 \mathrm{MHz}$ using TMS as internal standard. Mass spectroscopy data of the product of Suzuki reaction was collected on a MS-EI instrument. Infrared spectra were obtained from a FTIR spectrometer.

Except T4-8 and T4-14, which is new compound and thoroughly characterized by ${ }^{1} \mathrm{H}$ NMR, ${ }^{13} \mathrm{C}$ NMR, MS, HRMS and IR, the other prepared compounds are known and the analysis of ${ }^{1} \mathrm{H}$ NMR as well as MS for most compounds is performed. The MS data for T4-9, T4-10, T4-11 was very difficult to be collected, and for these three compounds, the elementary analysis was performed. In addition, the CA numbers as well as the related preparation and characterization reference are presented in Supporting Information.

General procedure for Suzuki reaction: A mixture of $\mathrm{Na}_{2} \mathrm{CO}_{3}(0.212 \mathrm{~g}, 2 \mathrm{mmol})$ (for dihalides, 4 mmol; for trihalides, $6 \mathrm{mmol}), \operatorname{Pd}(\mathrm{OAc})_{2}(1 \mathrm{mg}, 0.5 \mathrm{~mol} \%)$ (for dihalides, $\operatorname{Pd}(\mathrm{OAc})_{2}=1 \mathrm{~mol} \%$; for trihalides, $\left.\mathrm{Pd}(\mathrm{OAc})_{2}=1.5 \mathrm{~mol} \%\right)$, aryl halide $(1 \mathrm{mmol})$, arylboronic acid $(1.5 \mathrm{mmol})$ (for dihalides, boronic acid was $6 \mathrm{mmol}$; for trihalides, boronic acid was $9 \mathrm{mmol})$, distilled water $(3.5 \mathrm{~mL})$ and solvent $(3 \mathrm{~mL})$ was stirred for the indicated time. Afterward, the reaction solution was extracted four times with diethyl ether $(4 \times 10 \mathrm{~mL})$. The combined organic phase was analyzed by GC and GC/MS. The further purification of the product was achieved by flash chromatography on a silica gel column. 


\section{Characterization data of the product of the Suzuki reaction}

(1)

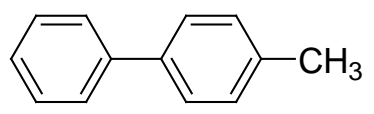

4-Methyl-biphenyl [T2-1, 644-08-6, Ref.1]

${ }^{1} \mathrm{H}$ NMR (500 MHz, $\mathrm{CDCl}_{3}$, TMS): $\delta 7.58(\mathrm{~d}, 2 \mathrm{H}, J=7.5 \mathrm{~Hz}), 7.49(\mathrm{~d}, 2 \mathrm{H}, J=8.0 \mathrm{~Hz}), 7.43(\mathrm{t}, 2 \mathrm{H}, J$

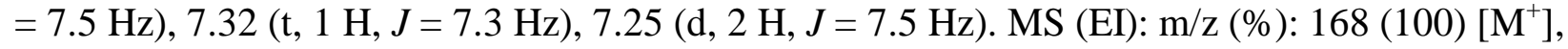
167(68), 165 (22), 152 (20), 115 (6).
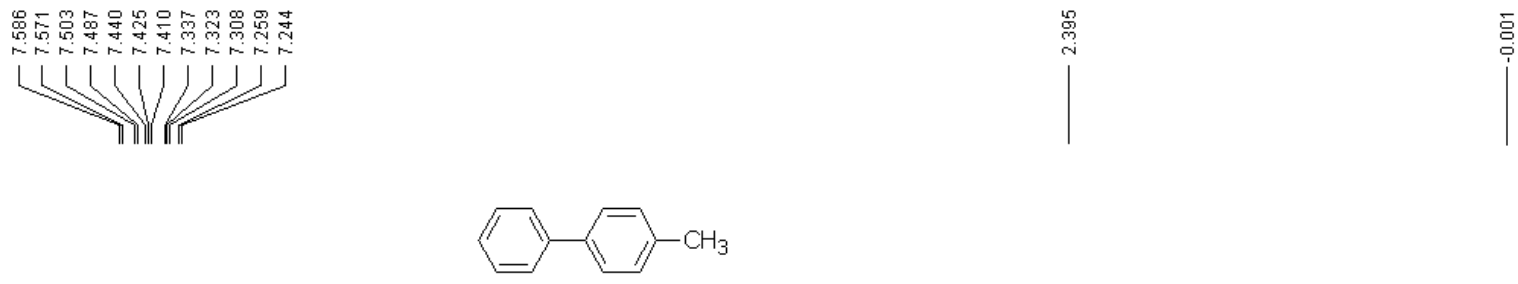

4-Methyl-biphenyl

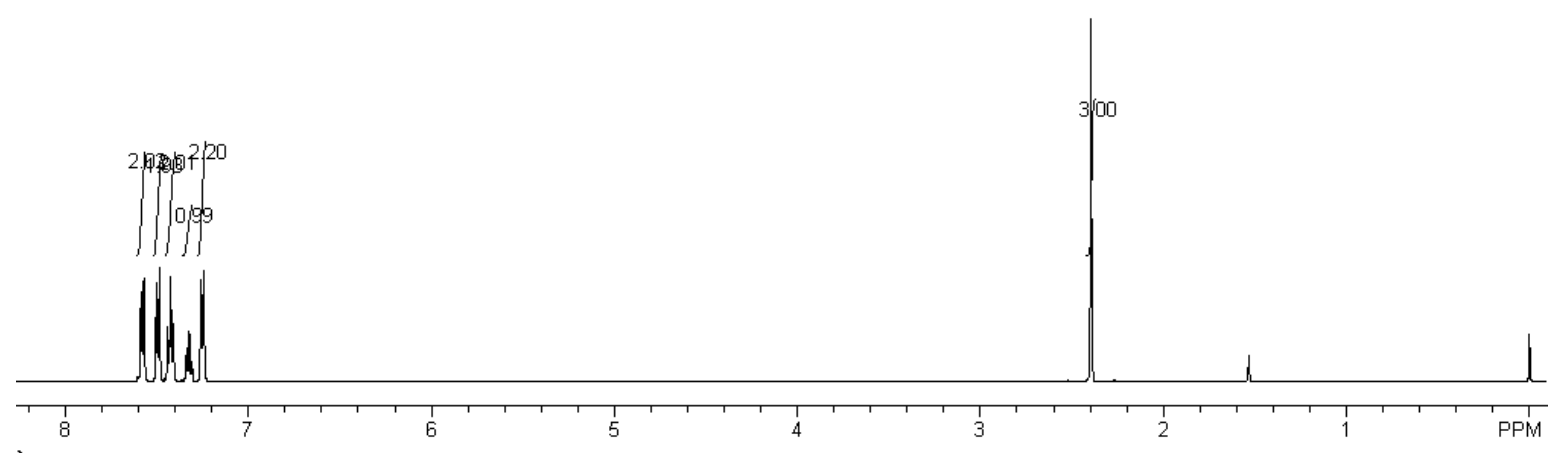


(2)

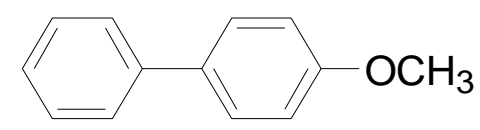

4-Methoxy-biphenyl [T2-2, 613-37-6, Ref. 1]

${ }^{1} \mathrm{H}$ NMR (500 MHz, CDCl 3 , TMS): $\delta 7.54(\mathrm{q}, 4 \mathrm{H}, J=6.7 \mathrm{~Hz}), 7.42(\mathrm{t}, 2 \mathrm{H}, J=7.7 \mathrm{~Hz}), 7.28(\mathrm{t}, 1 \mathrm{H}, J$

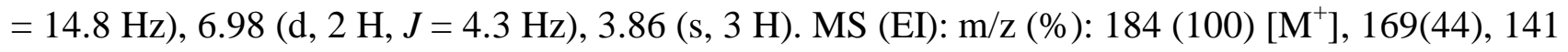
(38), 115 (26), 63 (4).

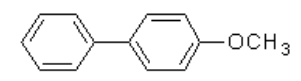

4-Methoxy-biphenyl

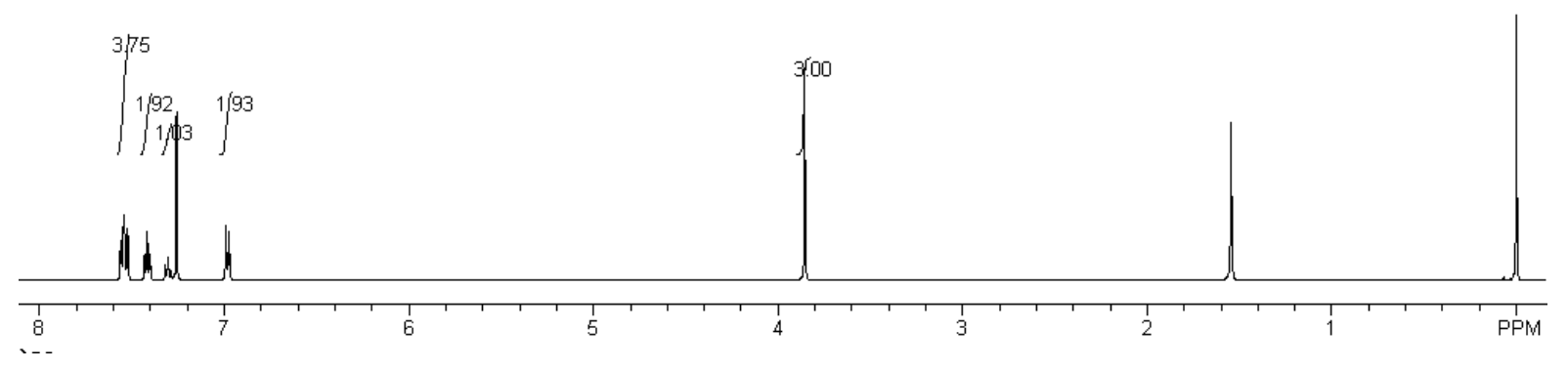


(3)

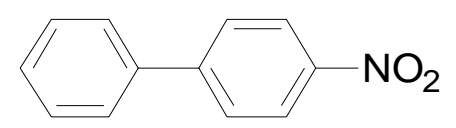

4-Nitro-biphenyl [T2-3, 92-93-3, Ref. 1]

${ }^{1} \mathrm{H}$ NMR (500 MHz, CDCl 3 , TMS): $\delta 8.32(\mathrm{~d}, 2 \mathrm{H}, J=9.0 \mathrm{~Hz}), 7.75(\mathrm{~d}, 2 \mathrm{H}, J=9.0 \mathrm{~Hz}), 7.64(\mathrm{~d}, 2 \mathrm{H}, J$

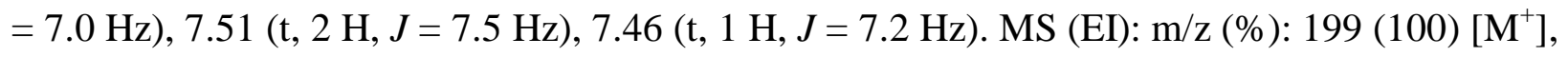
169(37), 152 (100), 141 (24), 115 (13), 76 (13), 63 (7), 51(6).
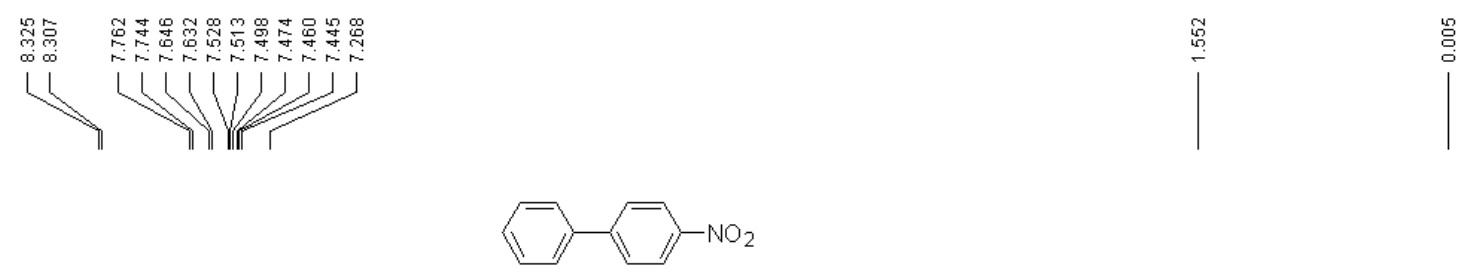

4-Nitro-biphenyl

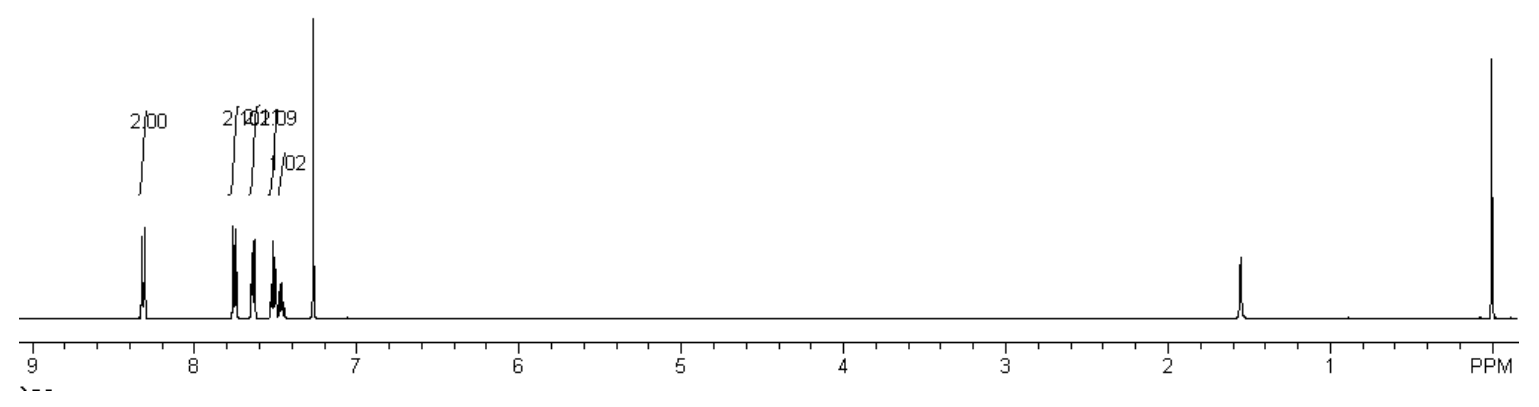




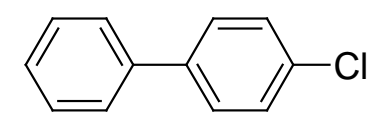

4-Chloro-biphenyl [T2-4, 2051-62-9, Ref. 2]

${ }^{1} \mathrm{H}$ NMR (500 MHz, $\mathrm{CDCl}_{3}$, TMS): $\delta 7.73(\mathrm{~d}, 1 \mathrm{H}, J=8.5 \mathrm{~Hz}), 7.69$ (d, $\left.2 \mathrm{H}, J=8.5 \mathrm{~Hz}\right), 7.65(\mathrm{~d}, 2 \mathrm{H}, J$ $=8.5 \mathrm{~Hz}), 7.52(\mathrm{t}, 2 \mathrm{H}, J=7.0 \mathrm{~Hz}), 7.44(\mathrm{t}, 2 \mathrm{H}, J=6.5 \mathrm{~Hz}) . \mathrm{MS}(\mathrm{EI}): \mathrm{m} / \mathrm{z}(\%): 188(100)\left[\mathrm{M}^{+}\right], 190$ (34) [M+2], 152(43), 76(15).
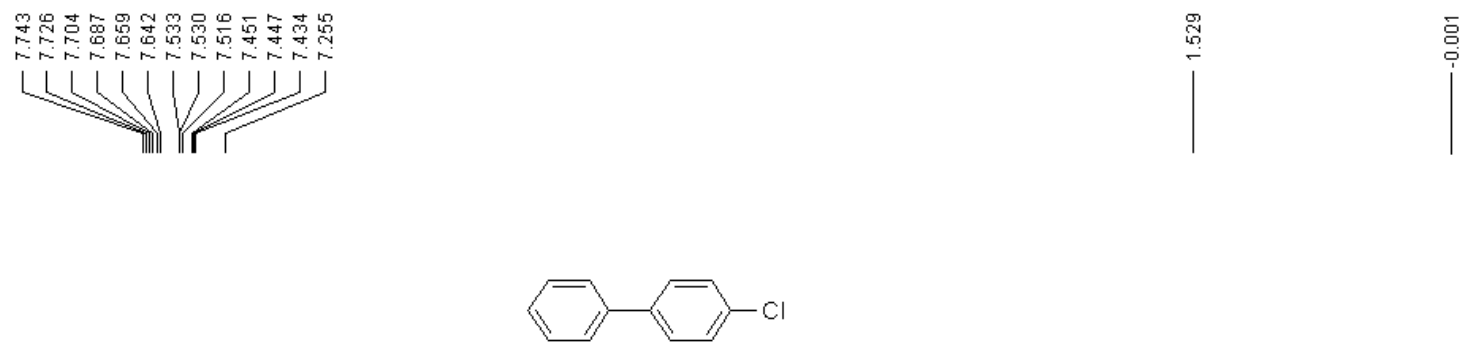

4-Chloro-biphenyl

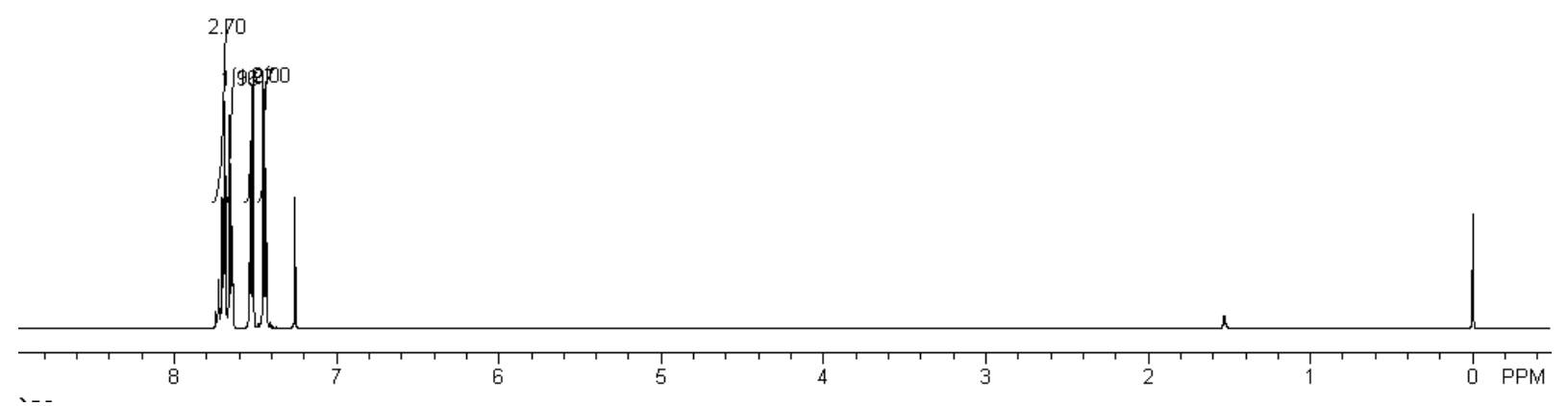




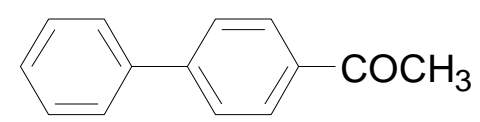

1-Biphenyl-4-yl-ethanone [T2-5, 92-91-1, Ref. 2]

${ }^{1} \mathrm{H}$ NMR (500 MHz, CDCl 3 , TMS): $\delta 8.03$ (d, $\left.2 \mathrm{H}, J=8.4 \mathrm{~Hz}\right), 7.69$ (d, $\left.2 \mathrm{H}, J=4.0 \mathrm{~Hz}\right), 7.63(\mathrm{t}, 2 \mathrm{H}, J$ $=4.5 \mathrm{~Hz}), 7.48(\mathrm{t}, 2 \mathrm{H}, J=7.5 \mathrm{~Hz}), 7.40(\mathrm{t}, 1 \mathrm{H}, J=7.0 \mathrm{~Hz}), 2.64$ (s, $3 \mathrm{H}) . \mathrm{MS}(\mathrm{EI}): \mathrm{m} / \mathrm{z}(\%): 196(51)$ $\left[\mathrm{M}^{+}\right], 181$ (100), 153(33), $152(51), 76(13), 43(4)$.
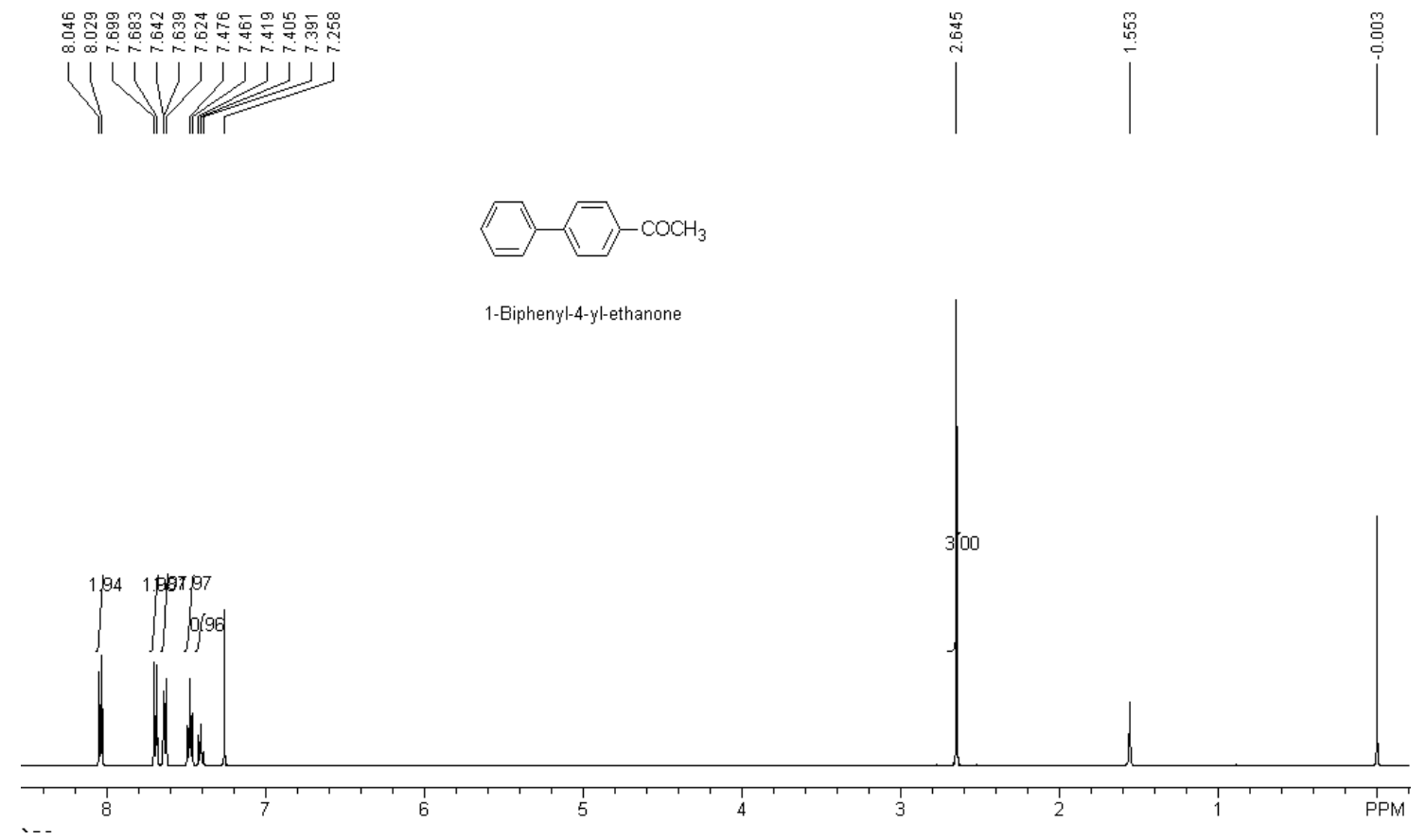


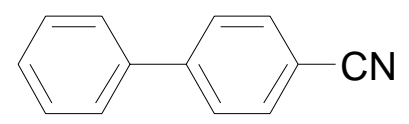

Biphenyl-4-carbonitrile [T2-6, 2920-38-9, Ref. 1]

${ }^{1} \mathrm{H}$ NMR (500 MHz, $\mathrm{CDCl}_{3}$, TMS): $\delta 7.73(\mathrm{~d}, 2 \mathrm{H}, J=8.5 \mathrm{~Hz}), 7.68(\mathrm{~d}, 2 \mathrm{H}, J=8.5 \mathrm{~Hz}), 7.59(\mathrm{t}, 2 \mathrm{H}, J$

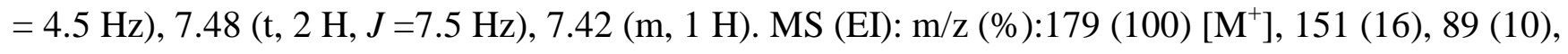
$76(14)$.
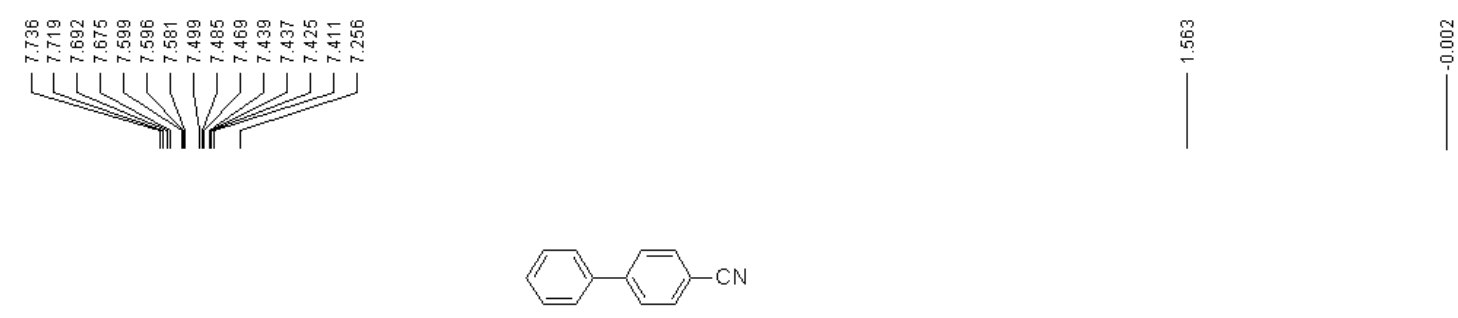

Biphenyl-4-carbonitrile

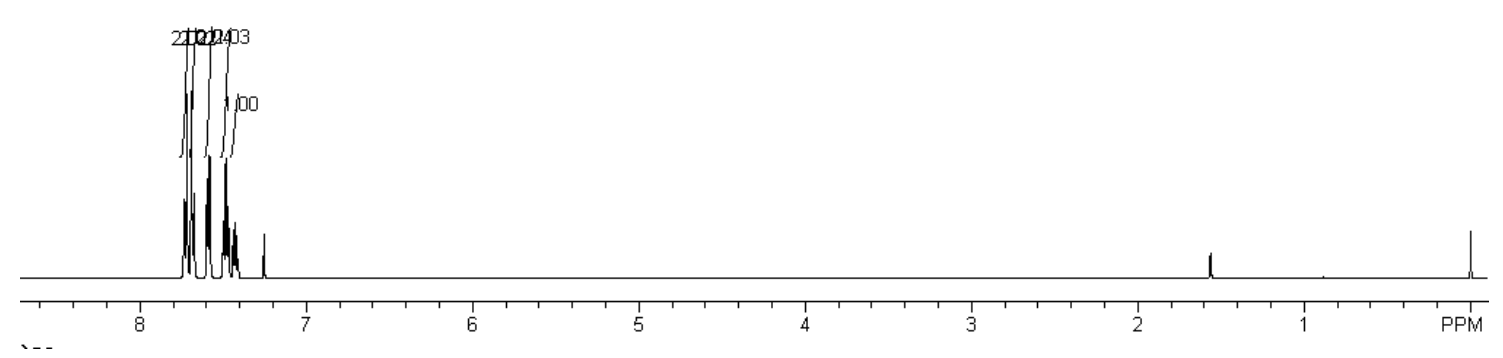


(7)

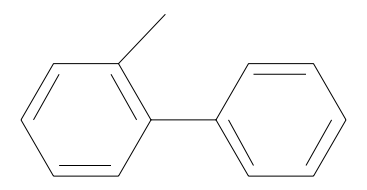

2-Methyl-biphenyl [T2-8, 643-58-3, Ref. 3]

${ }^{1} \mathrm{H}$ NMR (500 MHz, CDCl 3 , TMS): $\delta 7.42$ (t, $2 \mathrm{H}, J=7.5 \mathrm{~Hz}$ ), 7.34 (m, $\left.3 \mathrm{H}\right), 7.26$ (m, $4 \mathrm{H}$ ), 2.28 (s, 3 H). MS (EI): m/z (\%): 168 (100) [M+ $\mathrm{M}^{+}$, 167(100), 165 (60), 153 (59), 152(46), 141 (8), 139 (8), 115(14), 83(14), 63(6), 51(4).
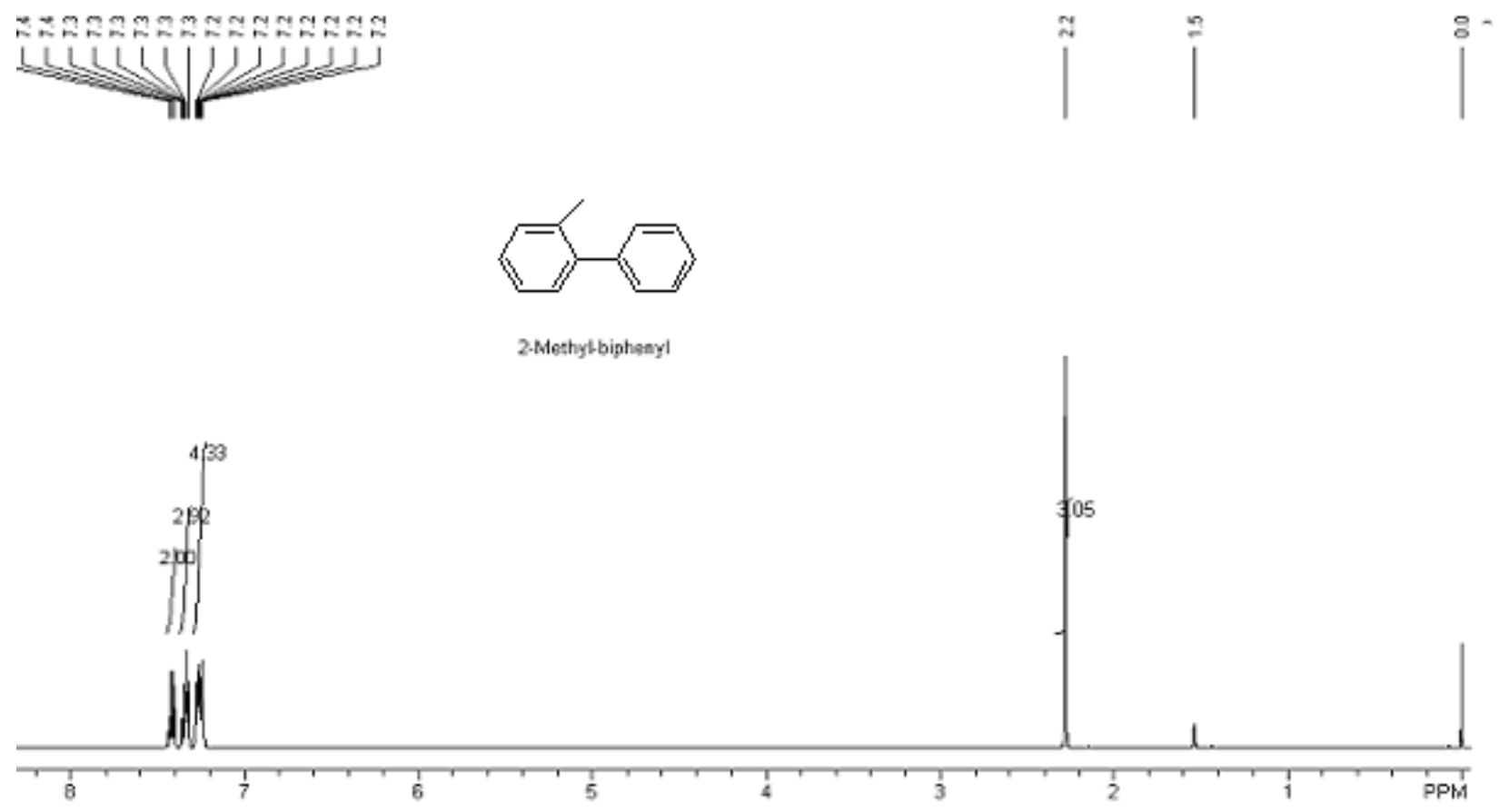
(8)

$\mathrm{HO}$

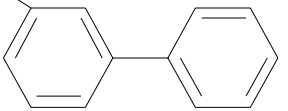

Biphenyl-3-ol [T2-9, 580-51-8, Ref. 4]

${ }^{1} \mathrm{H}$ NMR (500 MHz, $\left.\mathrm{CDCl}_{3}, \mathrm{TMS}\right): \delta 7.56(\mathrm{~d}, 2 \mathrm{H}, J=7.7 \mathrm{~Hz}), 7.43(\mathrm{t}, 2 \mathrm{H}, J=7.6 \mathrm{~Hz}), 7.34(\mathrm{~m}, 2 \mathrm{H})$, $7.17(\mathrm{~d}, 1 \mathrm{H}, J=7.7 \mathrm{~Hz}), 7.06(\mathrm{~s}, 1 \mathrm{H}), \quad 6.82(\mathrm{~m}, 1 \mathrm{H}), 4.82(\mathrm{~s}, 1 \mathrm{H}) \cdot \mathrm{MS}(\mathrm{EI}): \mathrm{m} / \mathrm{z}(\%): 170(100)\left[\mathrm{M}^{+}\right]$, $141(20), 115(14)$.
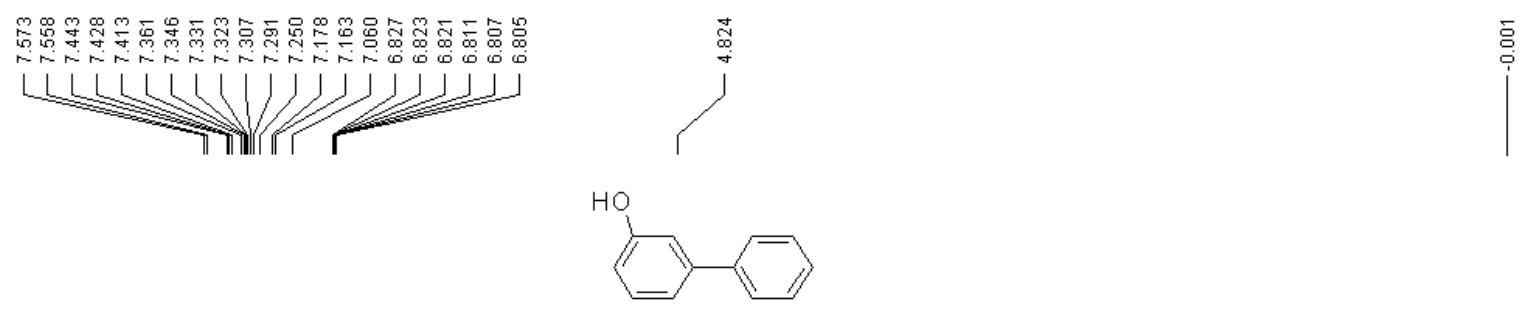

Biphenyl-3-ol

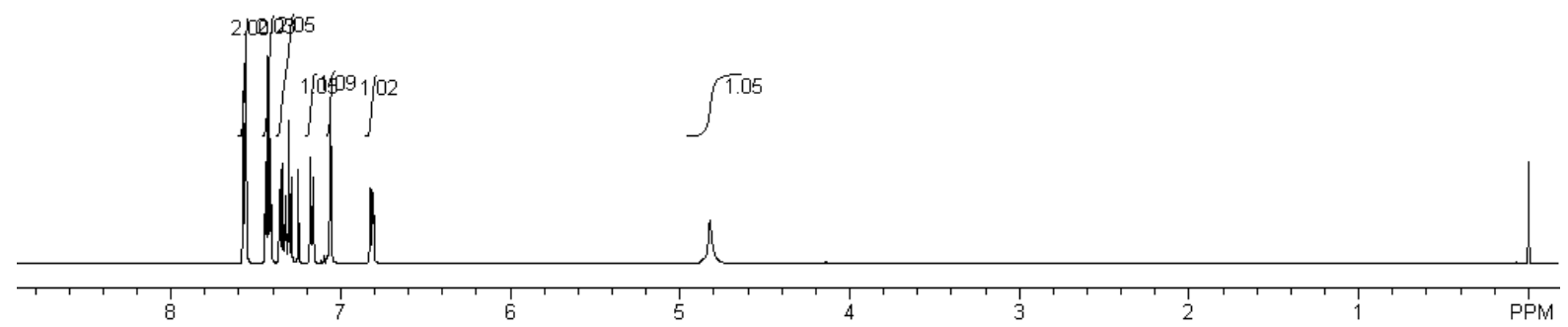




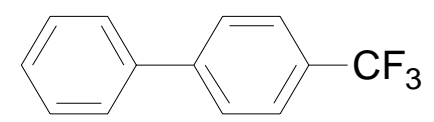

\section{4-Trifluoromethyl-biphenyl [T2-12, 398-36-7, Ref 5]}

${ }^{1} \mathrm{H}$ NMR (500 MHz, $\mathrm{CDCl}_{3}$, TMS): $\delta 7.69$ (s, $\left.4 \mathrm{H}\right), 7.60(\mathrm{~d}, 2 \mathrm{H}, J=8.0 \mathrm{~Hz}), 7.48(\mathrm{t}, 2 \mathrm{H}, J=7.5 \mathrm{~Hz})$, $7.41(\mathrm{t}, 1 \mathrm{H}, J=7.3 \mathrm{~Hz}), \mathrm{MS}(\mathrm{EI}): \mathrm{m} / \mathrm{z}(\%): 222(100)\left[\mathrm{M}^{+}\right], 201(13), 152(26), 86(4)$.
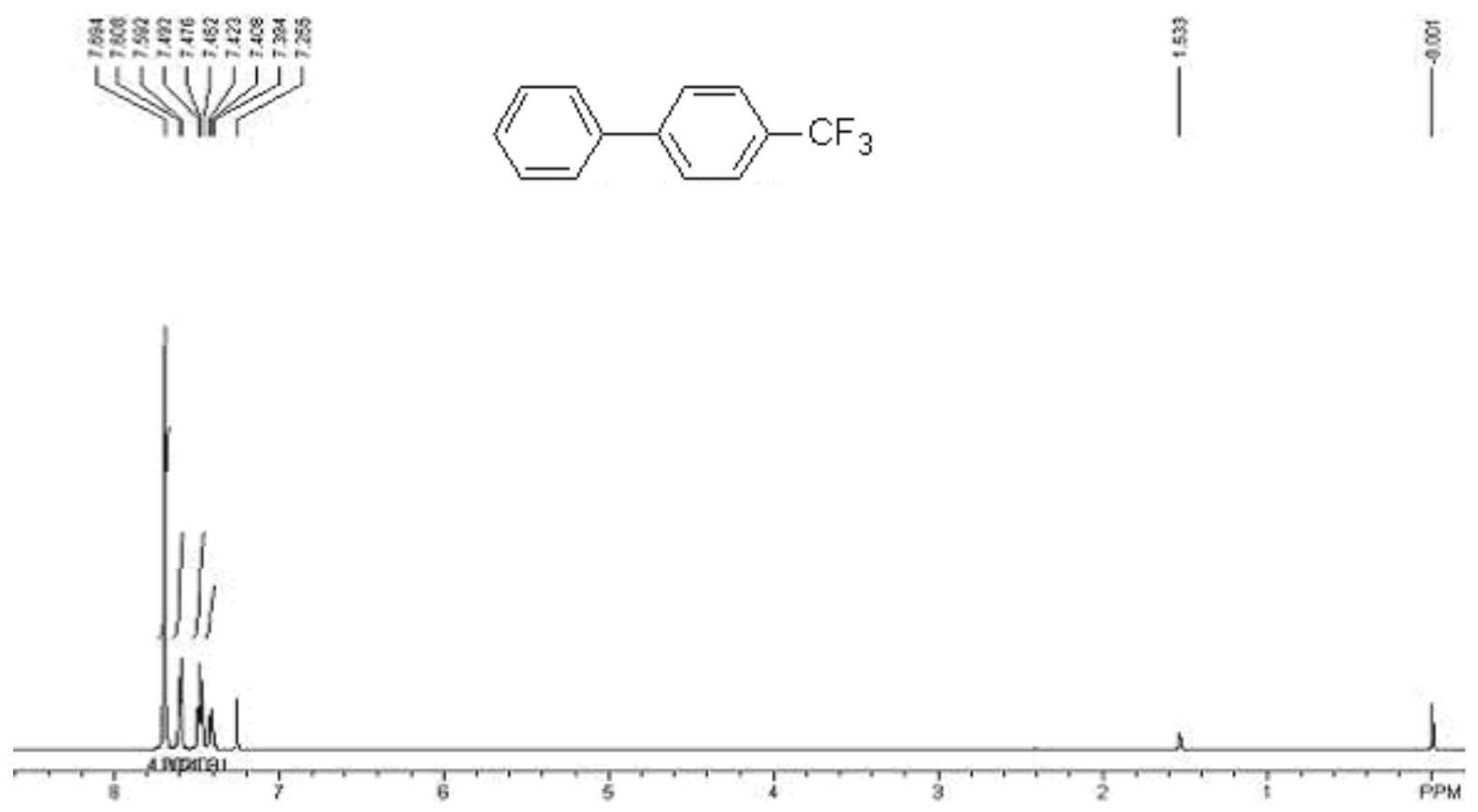


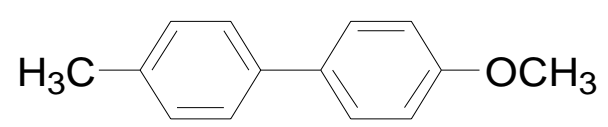

4-Methoxy-4'-methyl-biphenyl [T2-13, 53040-92-9, Ref 5]

${ }^{1} \mathrm{H}$ NMR (500 MHz, $\left.\mathrm{CDCl}_{3}, \mathrm{TMS}\right): \delta 7.51(\mathrm{~d}, 2 \mathrm{H}, J=8.0 \mathrm{~Hz}), 7.45(\mathrm{~d}, 2 \mathrm{H}, J=8.0 \mathrm{~Hz}), 7.22(\mathrm{~d}, 2 \mathrm{H}$,

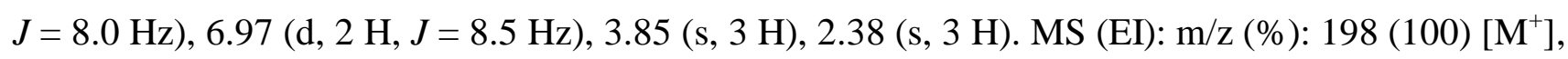
183 (54), 155 (27), $128(8), 115(5)$.
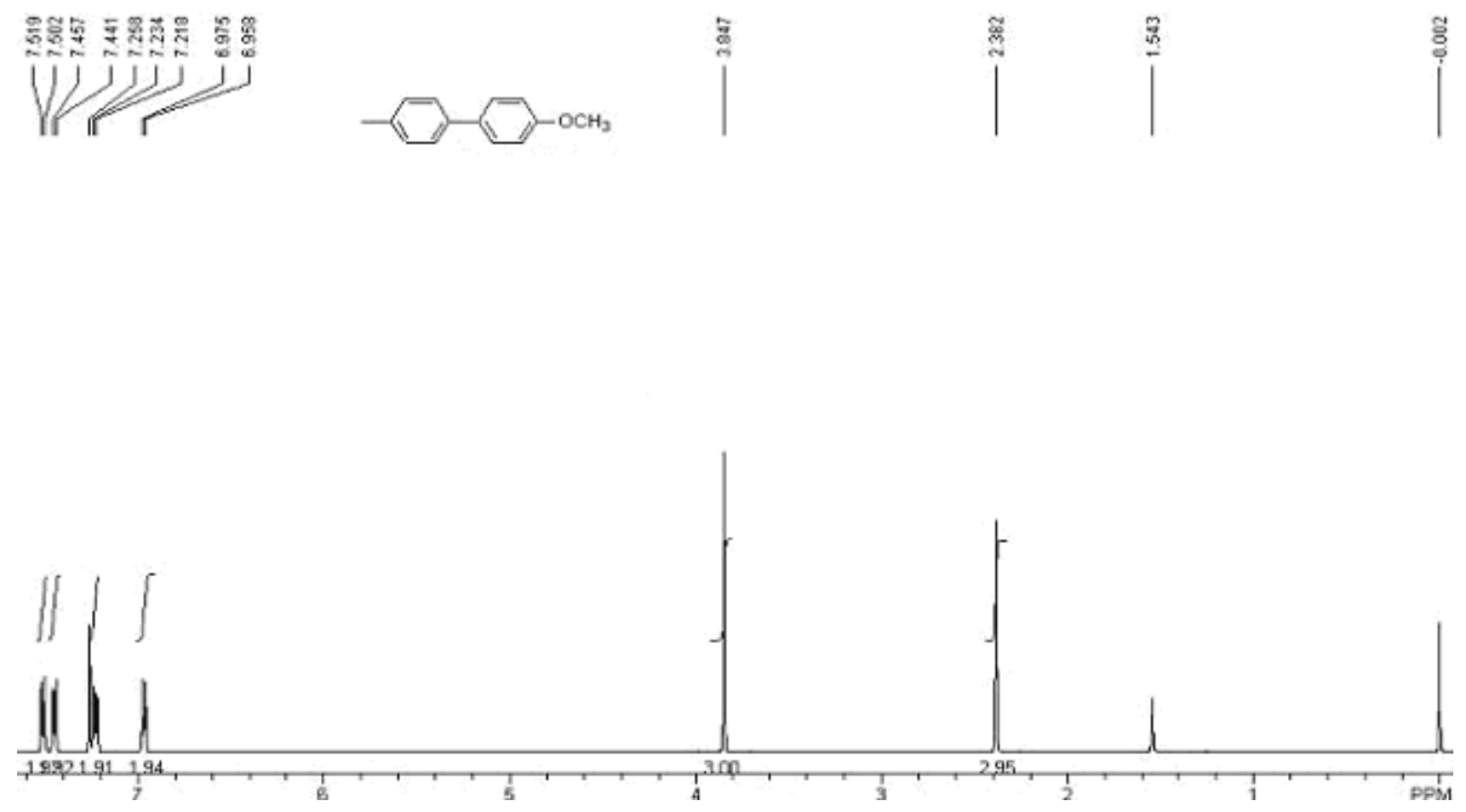


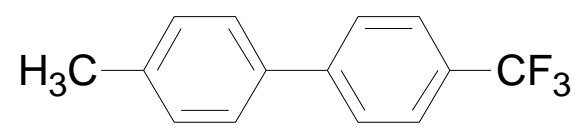

4'-Methyl-4-trifluoromethyl-biphenyl [T2-14, 97067-18-0, Ref. 6]

${ }^{1} \mathrm{H}$ NMR (500 MHz, $\mathrm{CDCl}_{3}$, TMS): $\delta 7.67$ (s, $\left.4 \mathrm{H}\right), 7.50(\mathrm{~d}, 2 \mathrm{H}, J=8.1 \mathrm{~Hz}), 7.28(\mathrm{~d}, 2 \mathrm{H}, J=8.0 \mathrm{~Hz})$, 2.40 (s, 3 H). MS (EI): m/z (\%): 236 (100) [M+1, 235 (25), 167 (38), 165(35), 152(8), 91 (7).
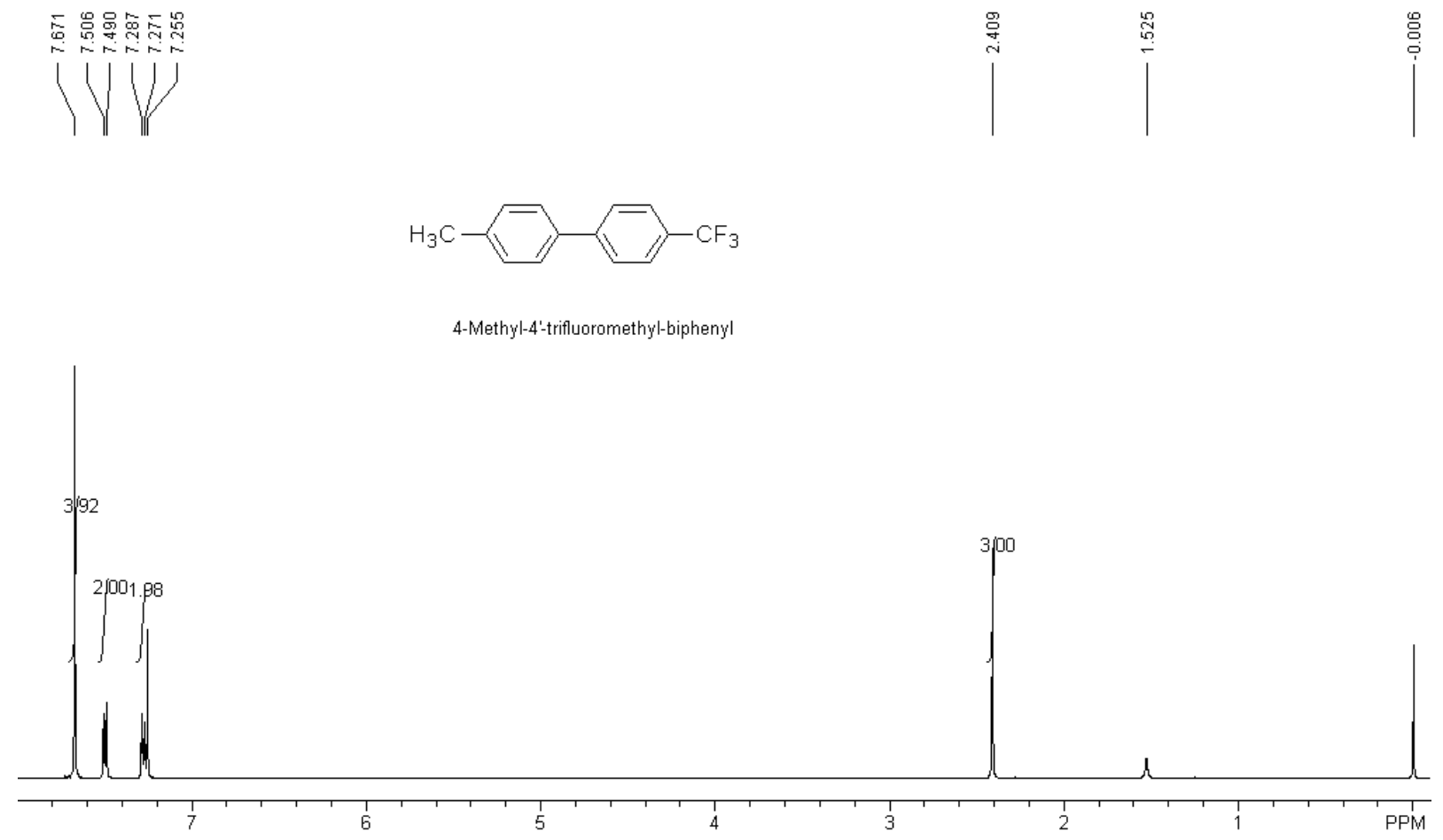


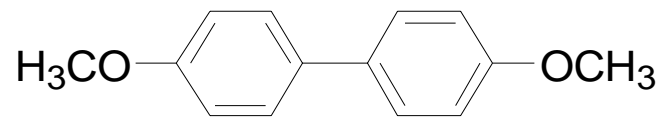

4, 4'-Dimethoxy-biphenyl [T2-15, 2132-80-1, Ref. 7]

${ }^{1} \mathrm{H}$ NMR (500 MHz, $\mathrm{CDCl}_{3}$, TMS): $\delta 7.52$ (d, $\left.4 \mathrm{H}, J=9.0 \mathrm{~Hz}\right), 7.00(\mathrm{~d}, 4 \mathrm{H}, J=8.5 \mathrm{~Hz}), 3.89(\mathrm{~s}, 6 \mathrm{H})$. MS (EI): m/z (\%): 214 (100) [M+1, 199 (81), 171 (24), 156 (8), 128 (13), 102 (16), 39 (10).
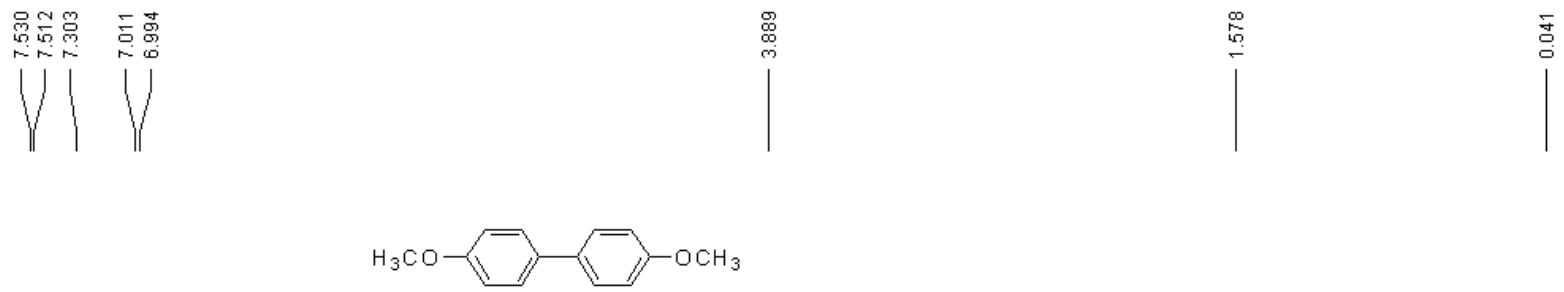

4,4'-Dimethoxy-biphenyl

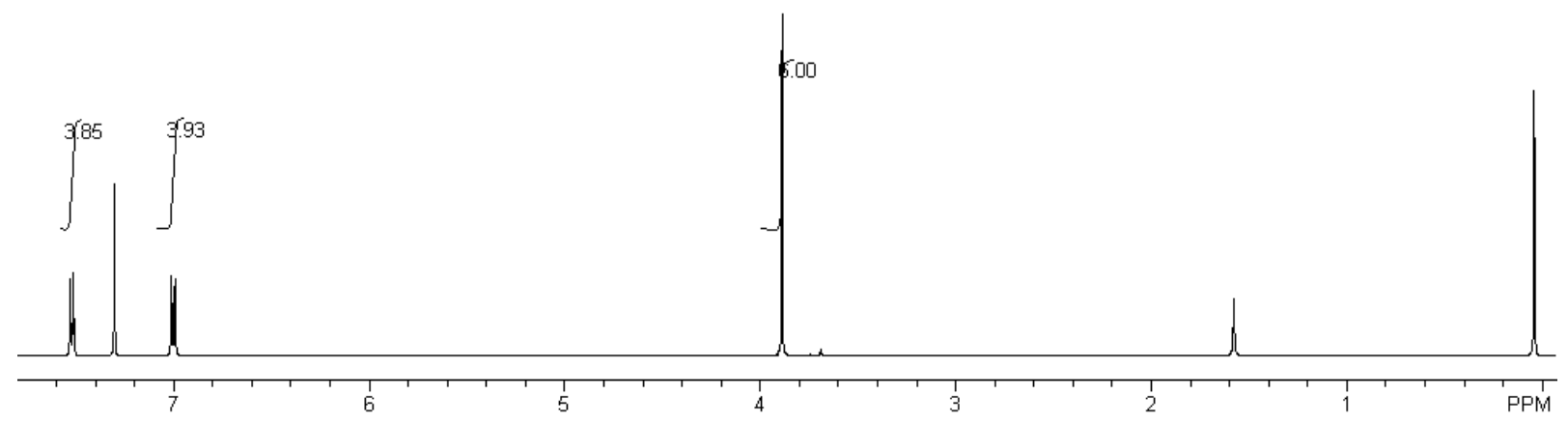


(13)

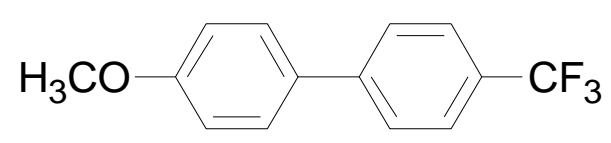

4-Methoxy-4'-trifluoromethyl-biphenyl [T2-16, 10355-12-1, Ref. 8]

${ }^{1} \mathrm{H}$ NMR (500 MHz, CDCl 3 , TMS): $\delta 7.65$ (q, $4 \mathrm{H}, J=9.0 \mathrm{~Hz}$ ), 7.54 (d, $\left.2 \mathrm{H}, J=8.7 \mathrm{~Hz}\right), 7.00(\mathrm{~d}, 2 \mathrm{H}, J$

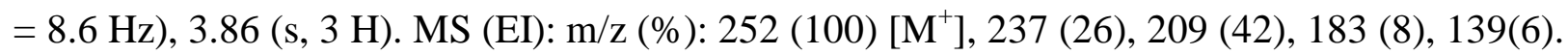
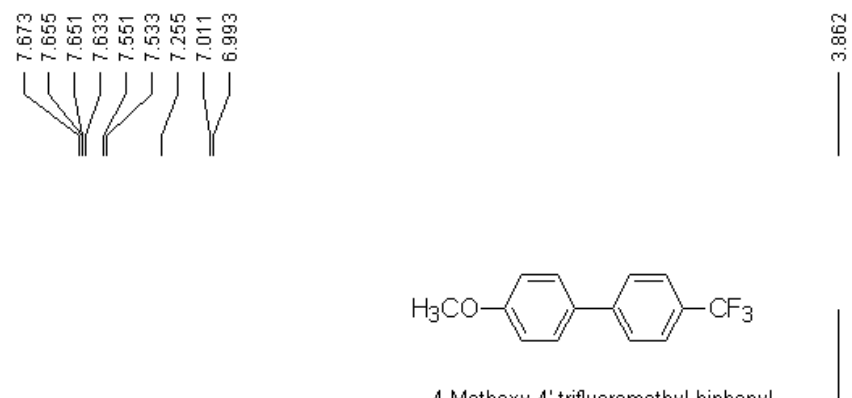

4-Methoxy-4'-trifluoromethyl-biphenyl

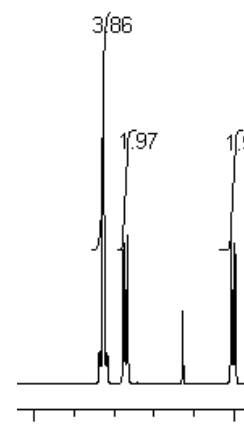

00

$$
\begin{gathered}
7 \\
8 \\
\hdashline-
\end{gathered}
$$

$\therefore-$ 


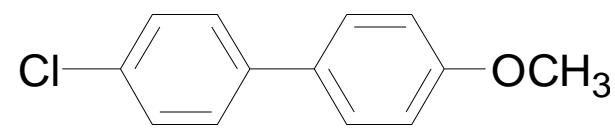

\section{4-Chloro-4'-methoxy-biphenyl [T2-17, 58970-19-7, Ref. 9]}

${ }^{1} \mathrm{H}$ NMR (500 MHz, $\mathrm{CDCl}_{3}$, TMS): $\delta 7.48(\mathrm{t}, 4 \mathrm{H}, J=8.5 \mathrm{~Hz}), 7.37(\mathrm{~d}, 2 \mathrm{H}, J=8.5 \mathrm{~Hz}), 6.9(\mathrm{~d}, 2 \mathrm{H}, J=$ $8.7 \mathrm{~Hz}), 3.85$ (s, $3 \mathrm{H})$. MS (EI): m/z (\%): 218 (100) [M+], 220 [M+2] (34), 203 (50),
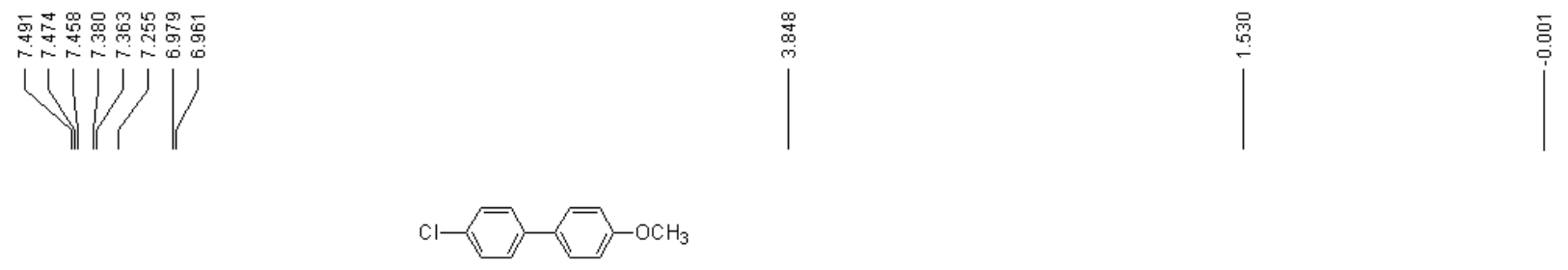

$$
\text { 4-Chloro-4'-methoxy-biphenyl }
$$

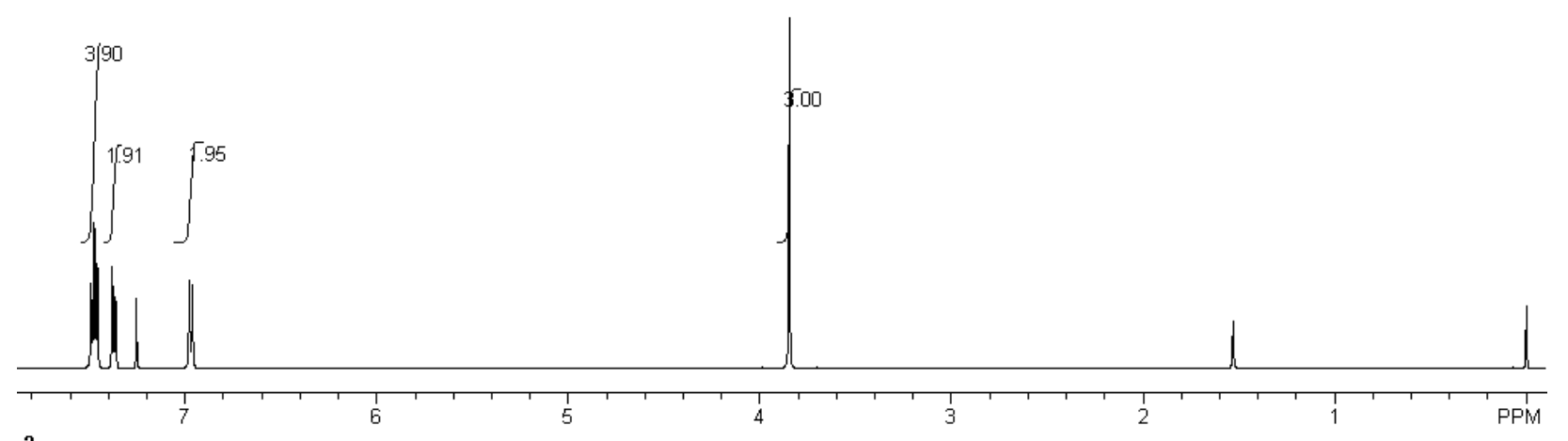




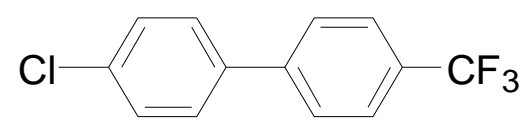

4-Chloro-4'-trifluoromethyl-biphenyl [T2-18, 146741-82-4, Ref. 10]

${ }^{1} \mathrm{H}$ NMR (500 MHz, CDCl 3 , TMS): $\delta 7.70(\mathrm{t}, 2 \mathrm{H}, J=8.5 \mathrm{~Hz}), 7.64(\mathrm{~d}, 2 \mathrm{H}, J=8.3 \mathrm{~Hz}), 7.53(\mathrm{t}, 2 \mathrm{H}, J$ $=6.9 \mathrm{~Hz}), 7.44$ (t, $3 \mathrm{H}, J=6.8 \mathrm{~Hz})$. MS (EI): m/z (\%): 256 (100) [M+1, 258[M+2] (34), 237(8), 201(14), 152(20).
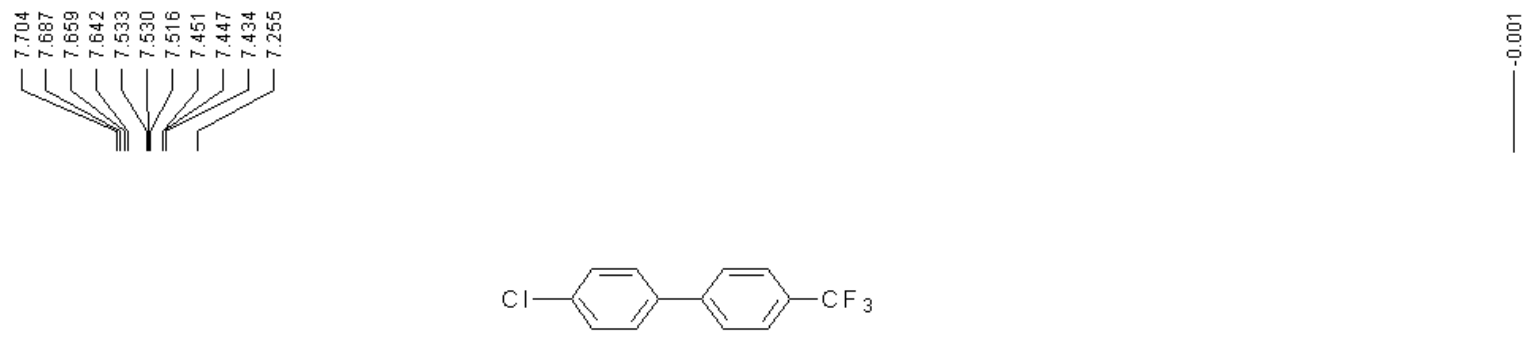

4-Chloro-4'-trifluoromethyl-biphenyl

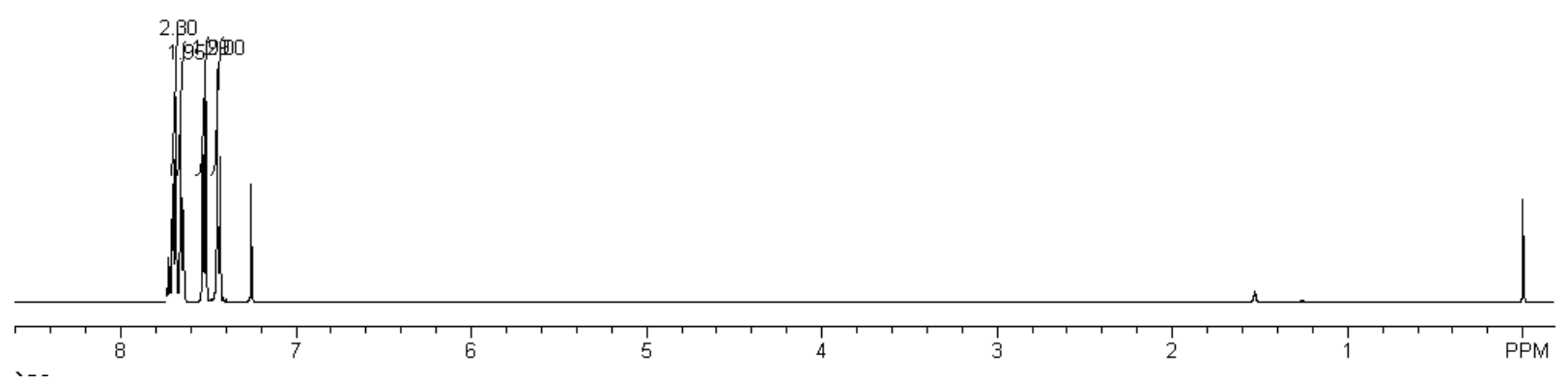




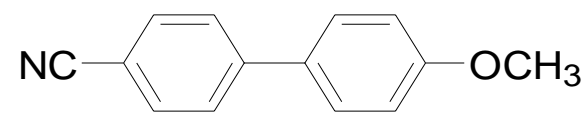

4'-Methoxy-biphenyl-4-carbonitrile [T2-19, 58743-77-4, Ref. 11]

${ }^{1} \mathrm{H}$ NMR (500 MHz, $\left.\mathrm{CDCl}_{3}, \mathrm{TMS}\right): \delta 7.69$ (d, $\left.2 \mathrm{H}, J=8.4 \mathrm{~Hz}\right), 7.64$ (d, $\left.2 \mathrm{H}, J=8.4 \mathrm{~Hz}\right), 7.54(\mathrm{~d}, 2 \mathrm{H}, J$

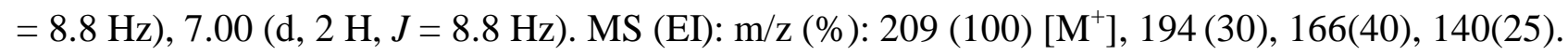
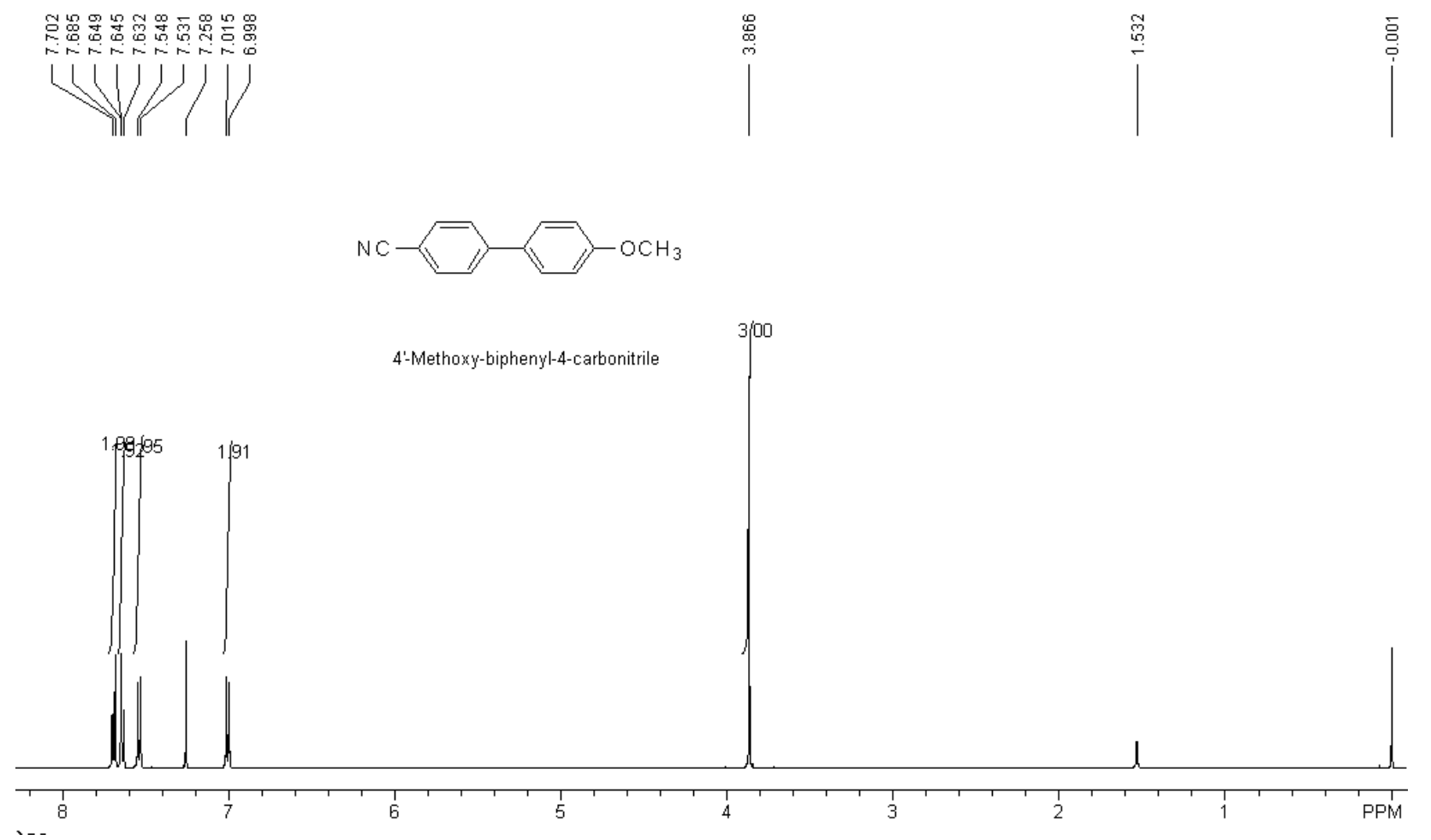


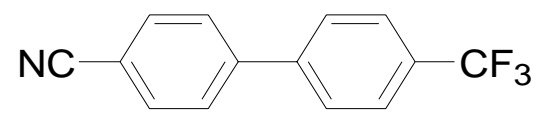

4'-Trifluoromethyl-biphenyl-4-carbonitrile [T2-20, 140483-60-9, Ref. 12]

${ }^{1} \mathrm{H}$ NMR (500 MHz, CDCl 3 , TMS): $\delta 7.76$ (m, 4 H), 7.70 (m, 4 H). MS (EI): m/z (\%): 247 (100) [M+ 228(12), 197(8), 177(10) 151(8).
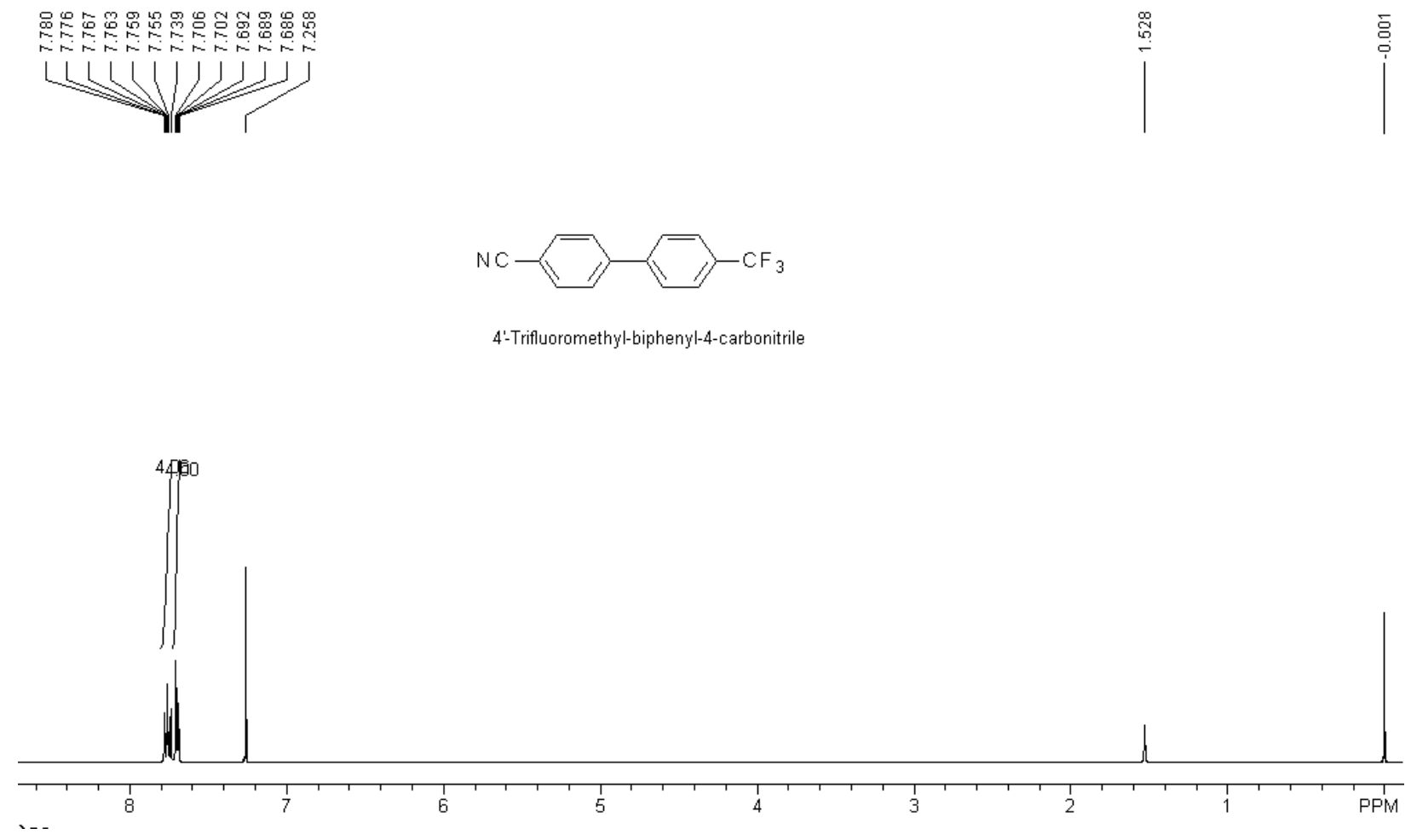


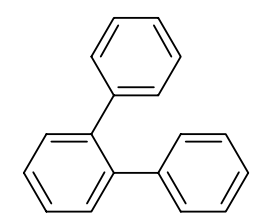

\section{1, 2-Diphenylbenzene [T4-1, 84-15-1, Ref. 13]}

${ }^{1} \mathrm{H}$ NMR (400 MHz, $\mathrm{CDCl}_{3}$, TMS): $\delta 7.41(\mathrm{~m}, 4 \mathrm{H}), 7.19(\mathrm{~m}, 6 \mathrm{H}), 7.13(\mathrm{~m}, 4 \mathrm{H}) . \mathrm{MS}(\mathrm{EI}): \mathrm{m} / \mathrm{z}(\%)$ : 230 (100).
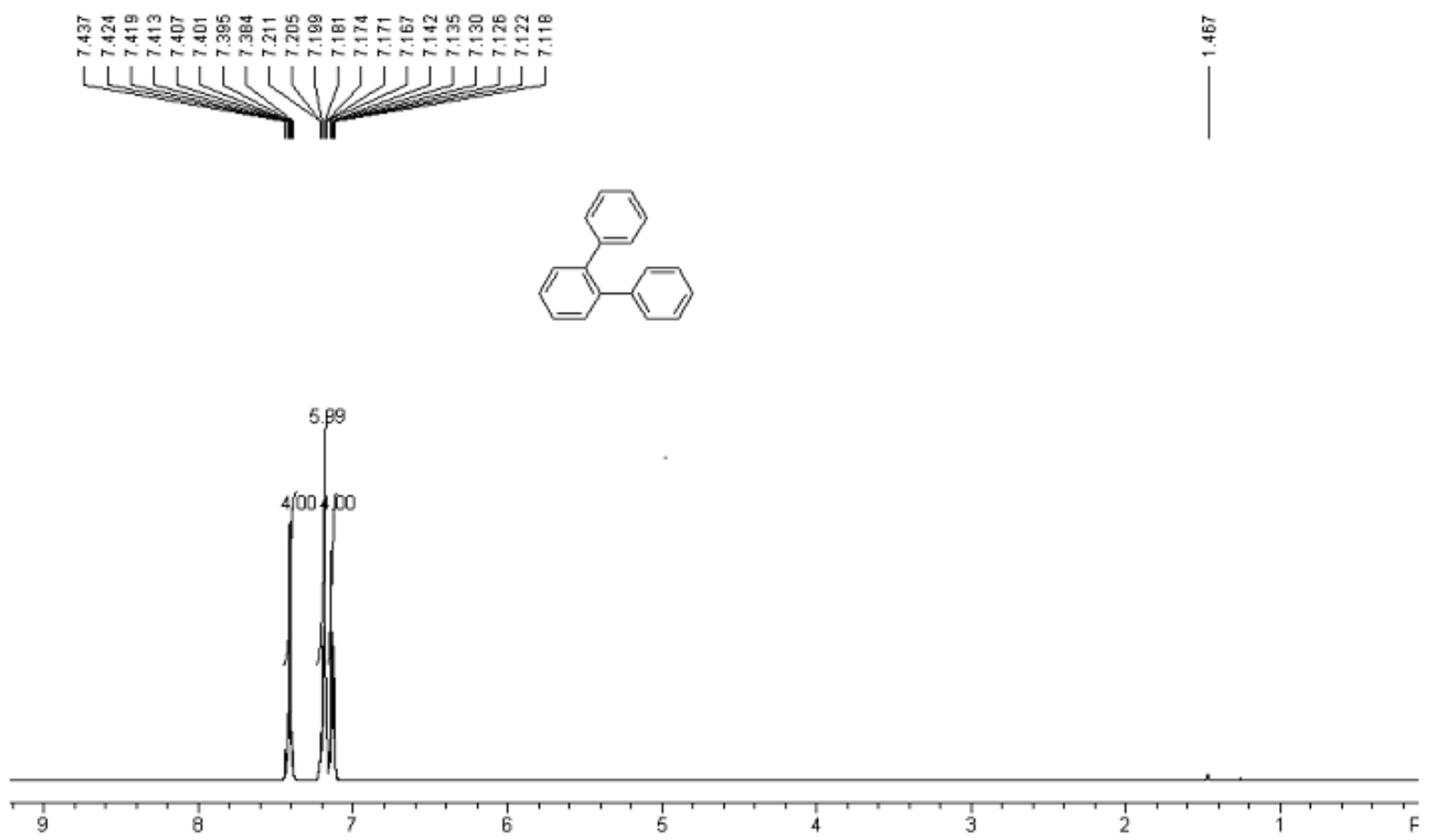


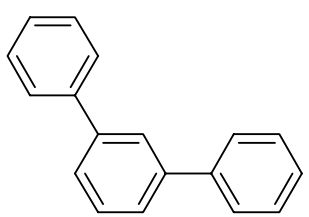

1, 3-Diphenylbenzene [T4-2, 92-06-8, Ref. 14]

${ }^{1} \mathrm{H}$ NMR (400 MHz, $\left.\mathrm{CDCl}_{3}, \mathrm{TMS}\right): \delta 7.83(\mathrm{~s}, 1 \mathrm{H}), 7.68(\mathrm{~d}, 4 \mathrm{H}, J=9.2 \mathrm{~Hz}), 7.61(\mathrm{~d}, 2 \mathrm{H}, J=8.4 \mathrm{~Hz})$, $7.54(\mathrm{~d}, 1 \mathrm{H}, J=7.4 \mathrm{~Hz}), 7.68(\mathrm{t}, 4 \mathrm{H}, J=7.6 \mathrm{~Hz}), 7.68$ (t, $2 \mathrm{H}, J=7.5 \mathrm{~Hz}) . \mathrm{MS}(\mathrm{EI}): \mathrm{m} / \mathrm{z}(\%): 230$ (100) $\left[\mathrm{M}^{+}\right], 202(10), 152(8), 115(12)$.
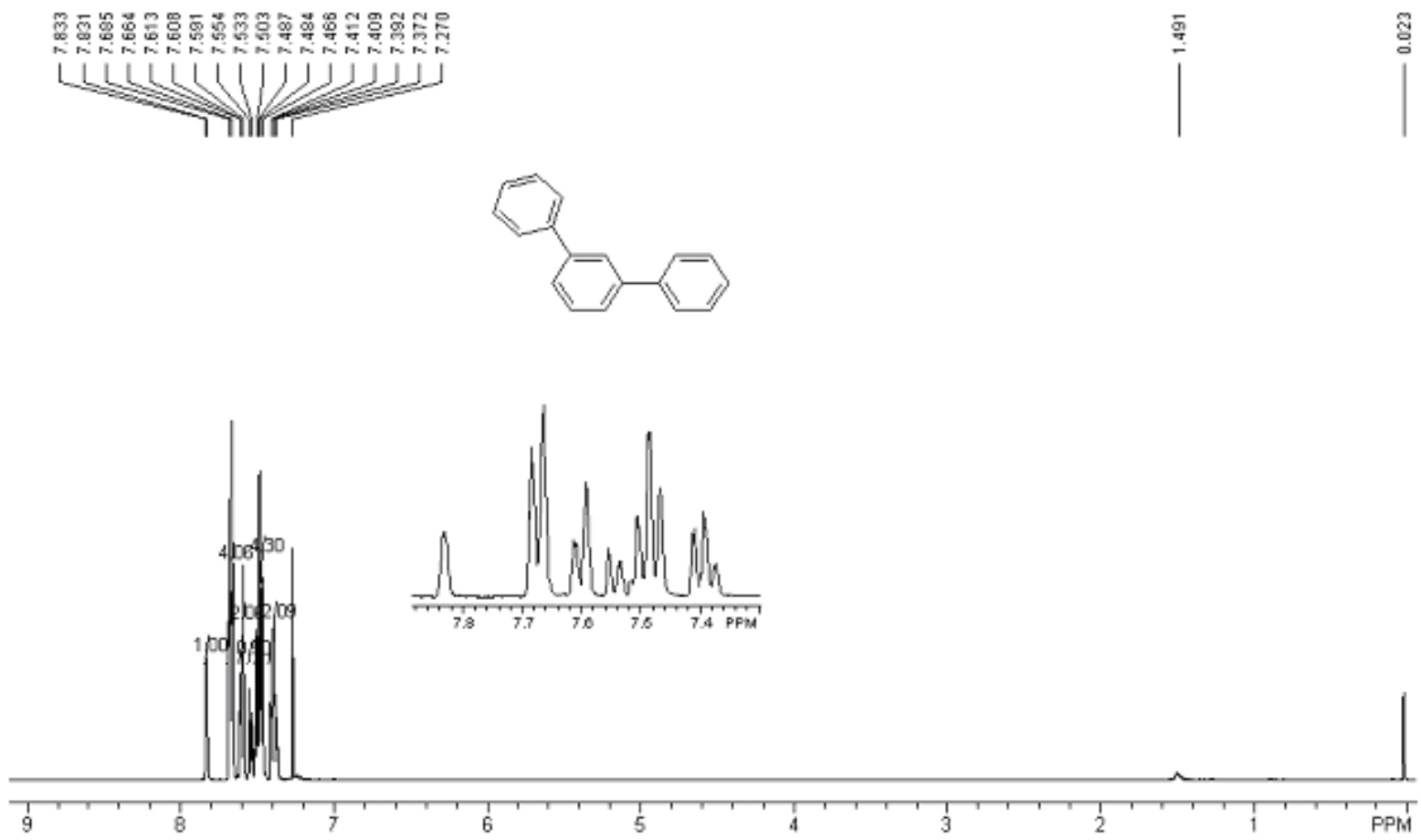


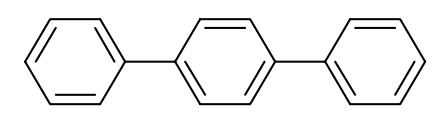

1, 4-Diphenylbenzene [T4-3, 92-94-4, Ref. 14]

${ }^{1} \mathrm{H}$ NMR (400 MHz, $\mathrm{CDCl}_{3}$, TMS): $\delta 7.68$ (s, $\left.4 \mathrm{H}\right), 7.68$ (d, $\left.4 \mathrm{H}, J=8.0 \mathrm{~Hz}\right), 7.46(\mathrm{t}, 4 \mathrm{H}, J=7.6 \mathrm{~Hz}$ ),

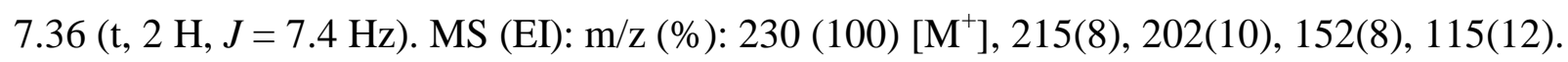
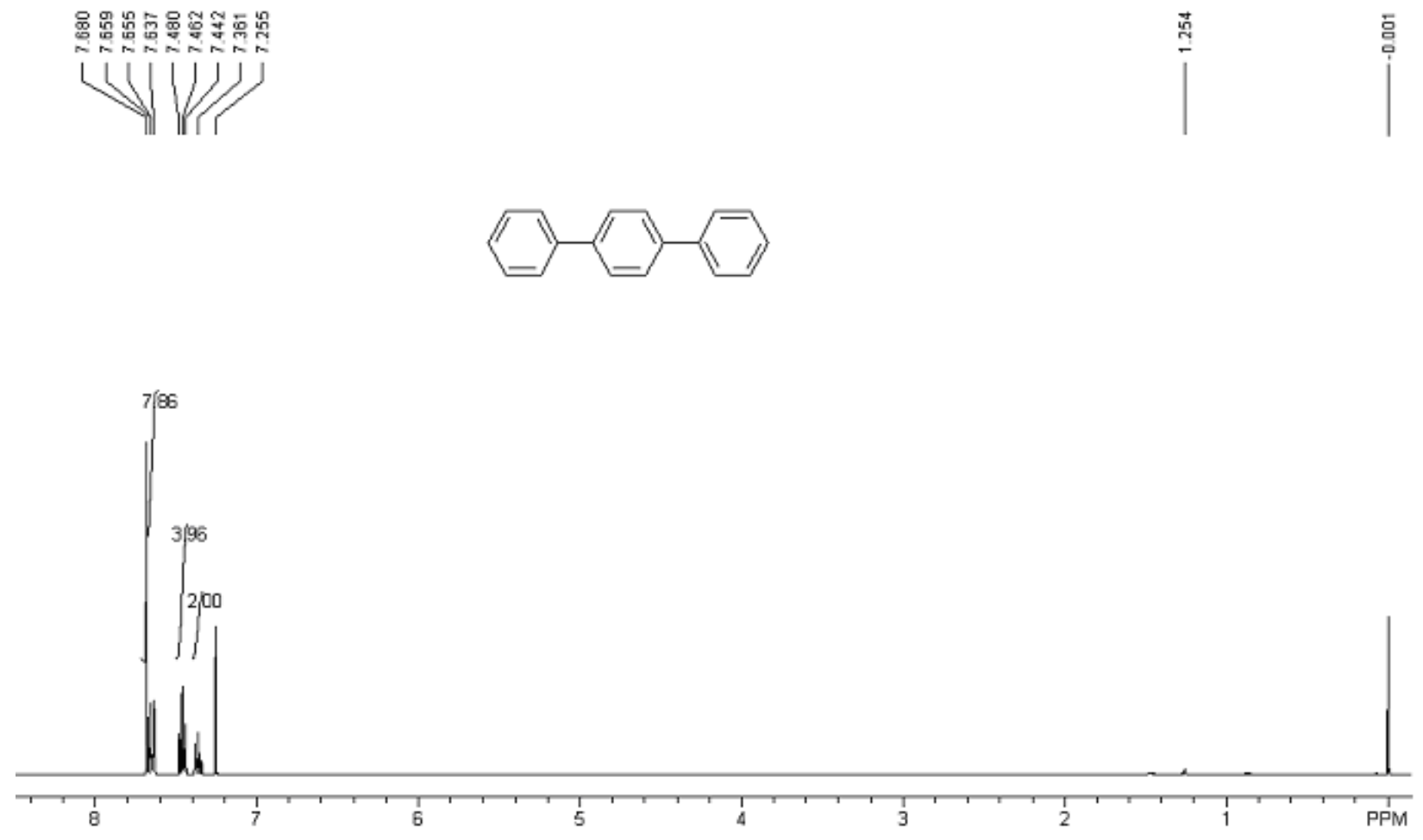


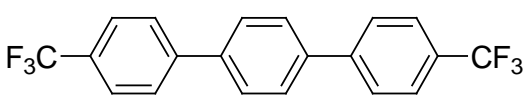

4, 4"'- Trifluoromethyl-1, 1': 4', 1"-terphenyl [T4-5, 72872-93-6, Ref. 15]

${ }^{1} \mathrm{H}$ NMR (400 MHz, $\left.\mathrm{CDCl}_{3}, \mathrm{TMS}\right): \delta$ 7.72-7.77 (m, $\left.6 \mathrm{H}\right) . \mathrm{MS}(\mathrm{EI}): \mathrm{m} / \mathrm{z}(\%): 366$ (100) [M $\left.{ }^{+}\right], 347(10)$, 296(8), 183(8), 158(5).
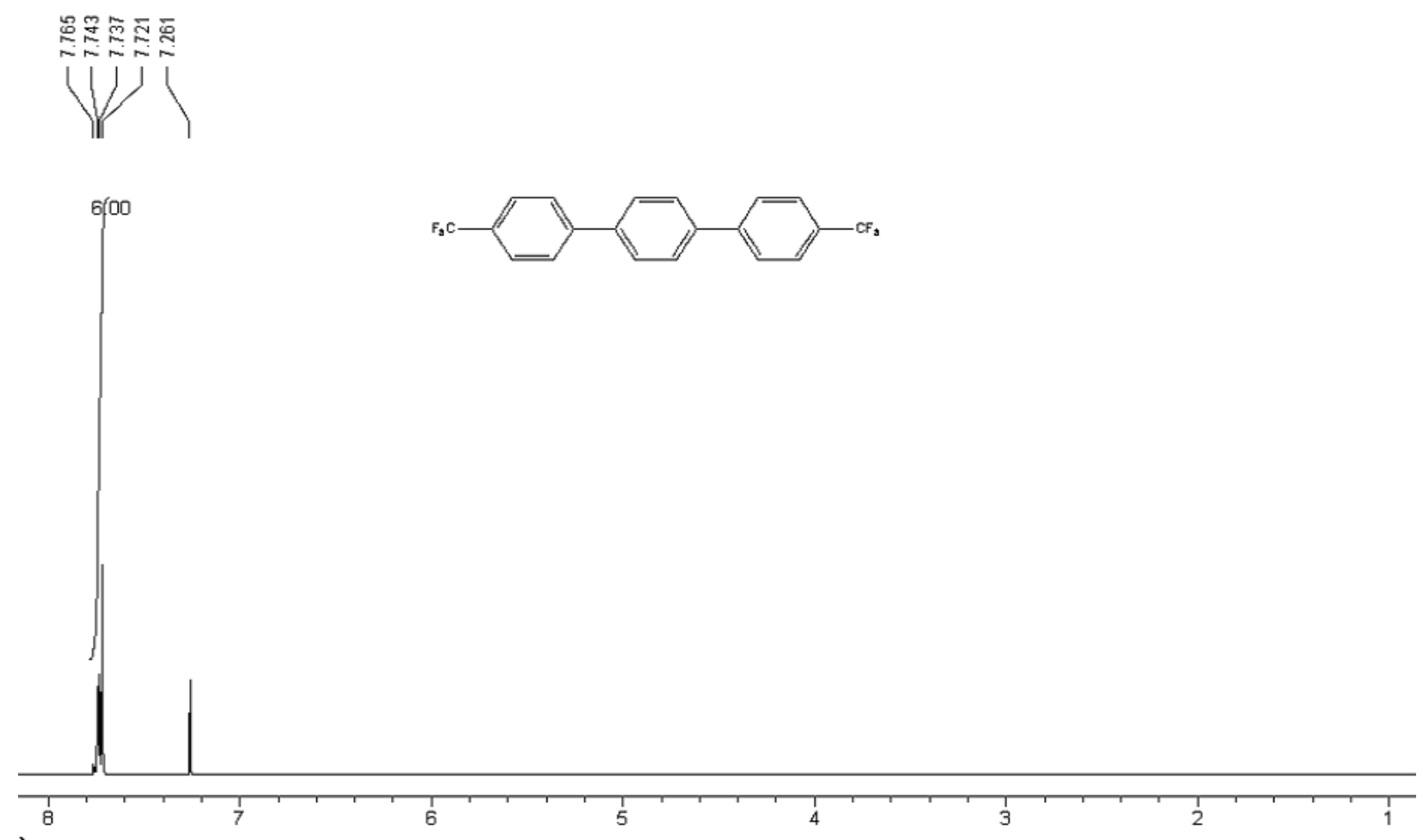
(22)

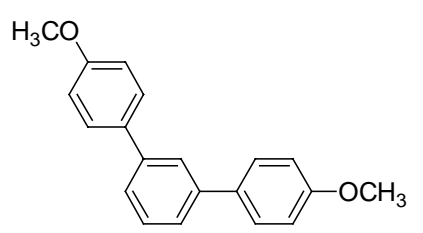

1, 3-Di(4-methoxyphenyl)benzene [T4-7, 1568-74-7, Ref. 14]

${ }^{1} \mathrm{H}$ NMR (500 MHz, $\mathrm{CDCl}_{3}$, TMS): $\delta 7.72(\mathrm{~s}, 1 \mathrm{H}), 7.58(\mathrm{~d}, 4 \mathrm{H}, J=8.7 \mathrm{~Hz}), 7.48(\mathrm{~m}, 3 \mathrm{H}), 7.00(\mathrm{~d}, 4 \mathrm{H}$,

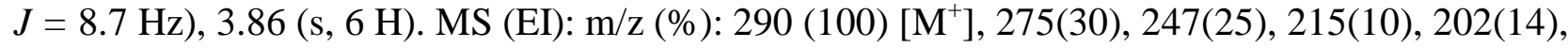
$145(15), 123(8)$.
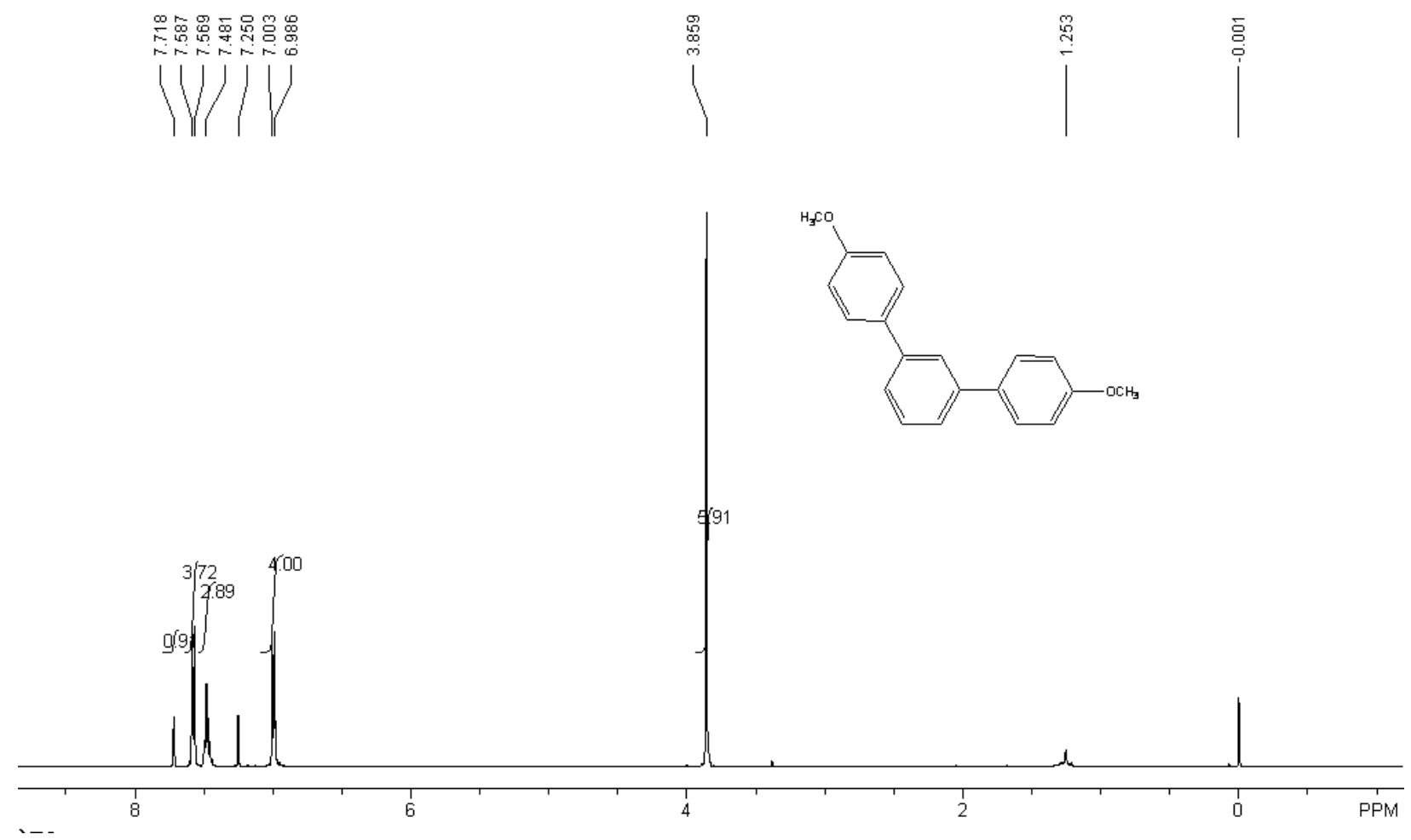


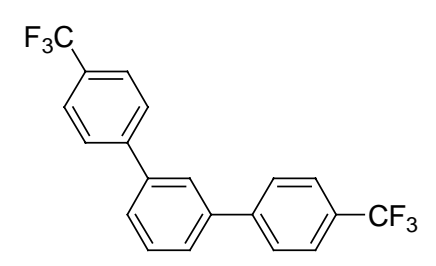

\section{4, 4"- Trifluoromethyl -1, 1':3', 1"-terphenyl [T4-8, New compound]}

${ }^{1} \mathrm{H}$ NMR (500 MHz, $\mathrm{CDCl}_{3}$, TMS): $\delta 7.79(\mathrm{~s}, 1 \mathrm{H}), 7.73(\mathrm{~m}, 8 \mathrm{H}), 7.64-7.56(\mathrm{~m}, 3 \mathrm{H}) ;{ }^{13} \mathrm{C}$ NMR $(125$ $\left.\mathrm{MHz}, \mathrm{CDCl}_{3}\right) \delta 144.6,140.9,130.0(\mathrm{q}, J=32.3 \mathrm{~Hz}), 129.9,127.8,127.4,126.6,126.1(\mathrm{q}, J=3.8 \mathrm{~Hz})$, $124.5(\mathrm{q}, J=270.3 \mathrm{~Hz})$; IR $(\mathrm{KBr}) v 1616,1573,1482,1410,1389,1323,1249,1173,1126,1070,1059$, 1023, 844, 796, 755, 737, 698, 601, $430 \mathrm{~cm}^{-1} . \mathrm{MS}(\mathrm{EI}): \mathrm{m} / \mathrm{z}(\%): 366$ (100) $\left[\mathrm{M}^{+}\right], 347(12), 296(10)$, 228(10), 183(8); HRMS (EI) calcd for $\mathrm{C}_{20} \mathrm{H}_{12} \mathrm{~F}_{6}, 366.0838$; found, 366.0835.

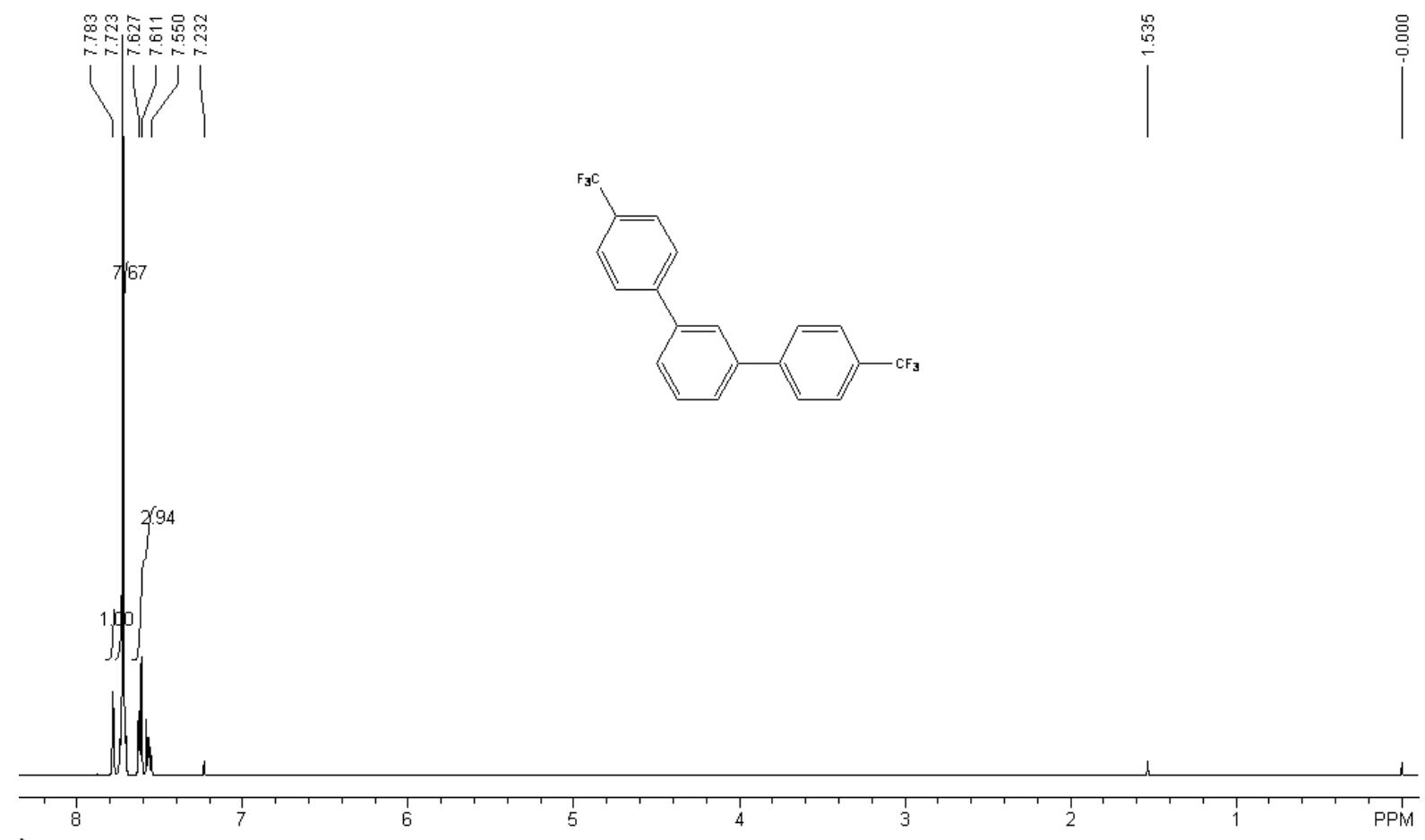



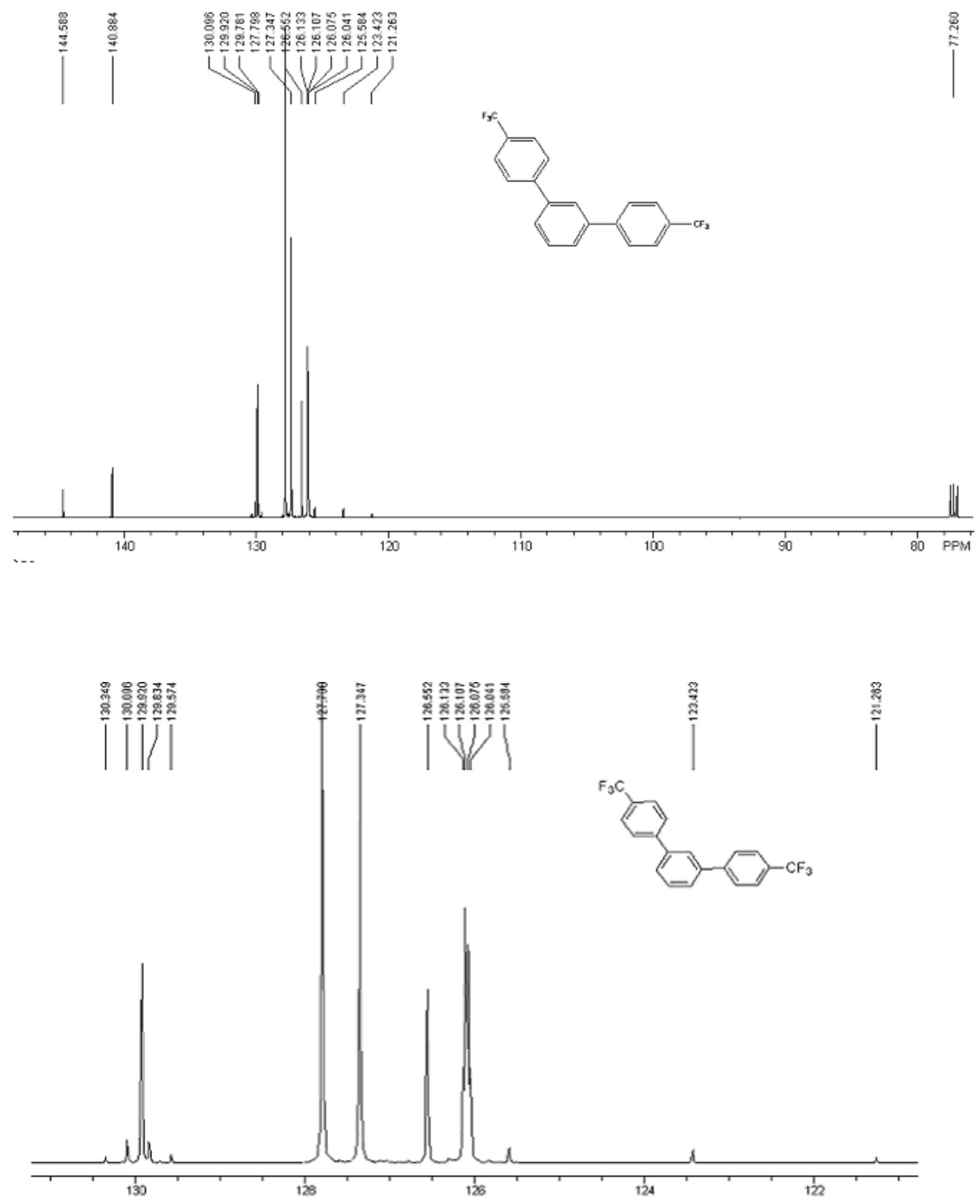


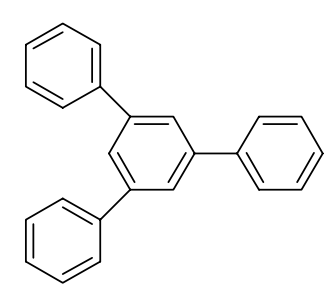

\section{1, 3, 5-Triphenylbenzene [T4-9, 612-71-5, Ref. 13]}

${ }^{1} \mathrm{H}$ NMR (400 MHz, CDCl , TMS): $\delta 7.79$ (s, $3 \mathrm{H}$ ), 7.68 (d, $\left.6 \mathrm{H}, J=8.4 \mathrm{~Hz}\right), 7.48(\mathrm{t}, 6 \mathrm{H}, J=7.2 \mathrm{~Hz}$ ),

$7.39(\mathrm{t}, 3 \mathrm{H}, J=7.4 \mathrm{~Hz}) ;{ }^{13} \mathrm{C} \mathrm{NMR}\left(125 \mathrm{MHz}, \mathrm{CDCl}_{3}\right) \delta 142.6,141.4,129.1,127.8,127.6,125.4$;

Anal. Calcd for $\mathrm{C}_{24} \mathrm{H}_{18}$ : C, 94.08; H, 5.92. Found: C, 94.12; H, 5.91.

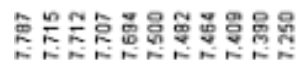

ivijij

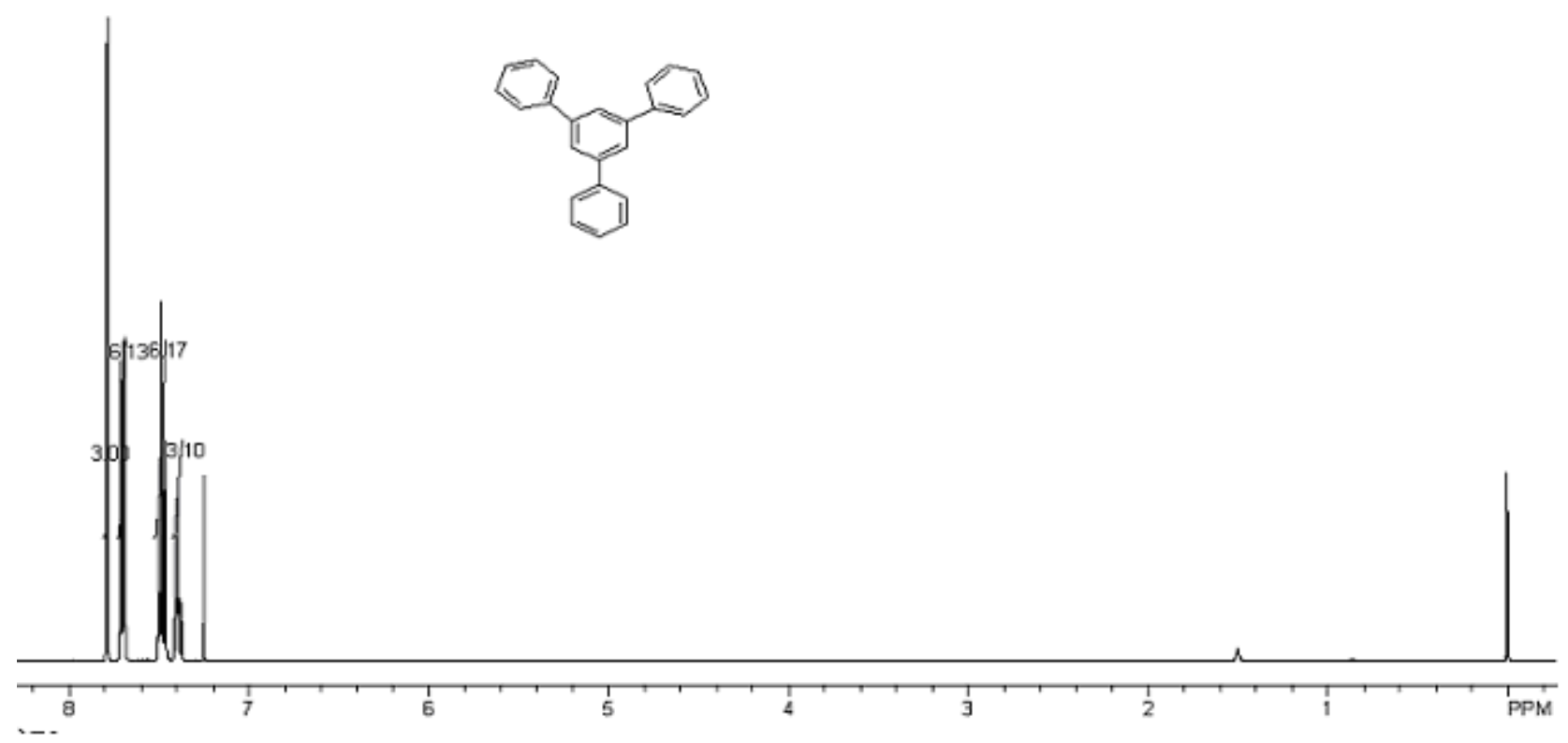




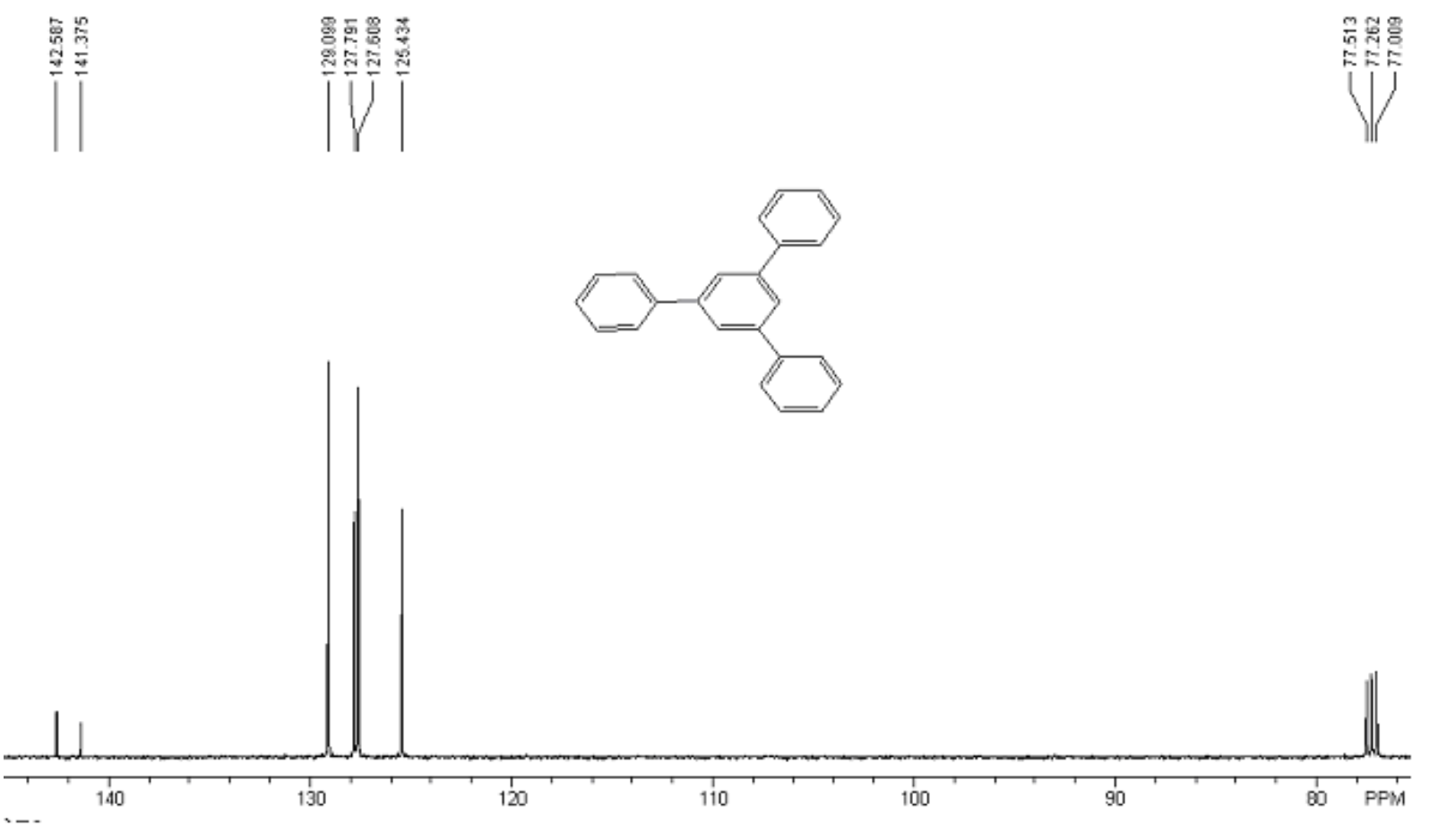

(25)

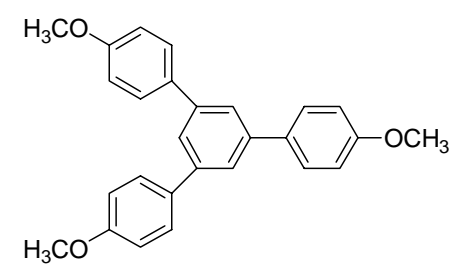

1, 3, 5-Tris(4-methoxyphenyl)benzene [T4-10, 7509-20-8, Ref. 16]

${ }^{1} \mathrm{H}$ NMR (500 MHz, $\mathrm{CDCl}_{3}$, TMS): $\delta 7.65$ (s, $\left.3 \mathrm{H}\right), 7.62(\mathrm{~d}, 6 \mathrm{H}, J=8.5 \mathrm{~Hz}), 7.01(\mathrm{~d}, 6 \mathrm{H}, J=9.0 \mathrm{~Hz})$, 3.87 (s, $9 \mathrm{H}) ;{ }^{13} \mathrm{C}$ NMR $\left(125 \mathrm{MHz}, \mathrm{CDCl}_{3}\right) \delta 159.5,142.1,134.1$, 128.6, 124.0, 114.5, 55.6; Anal. Calcd for $\mathrm{C}_{27} \mathrm{H}_{24} \mathrm{O}_{3}: \mathrm{C}, 81.79 ; \mathrm{H}, 6.10$. Found: $\mathrm{C}, 81.83 ; \mathrm{H}, 6.08$. 


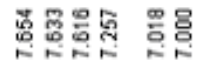

ivi
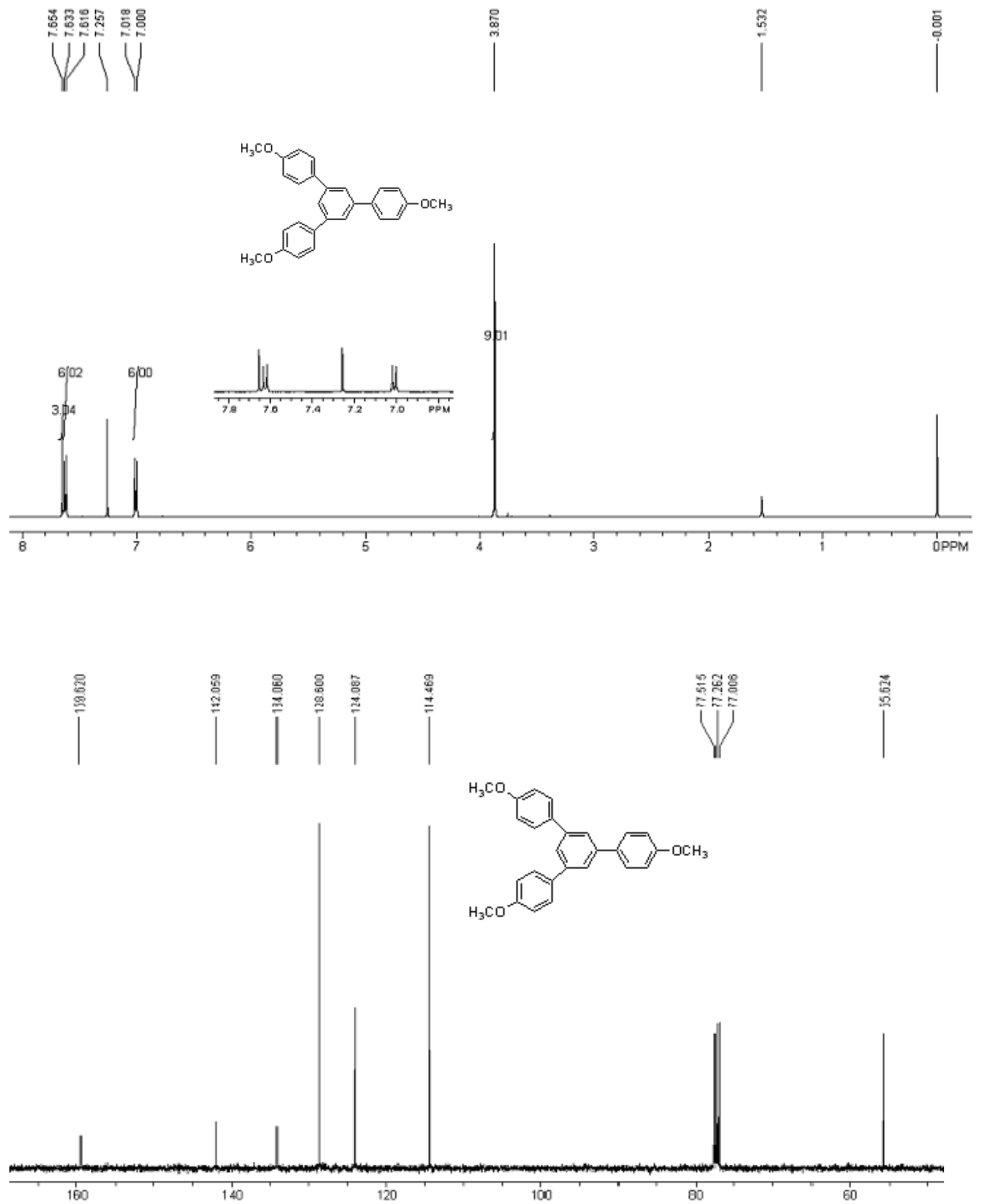
(26)

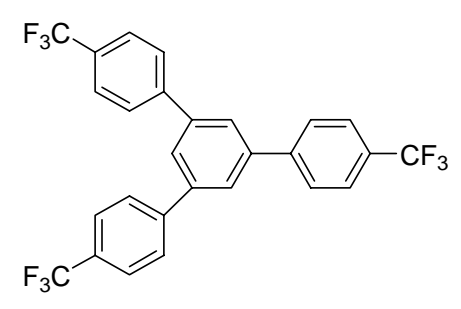

1, 3, 5-Tris(4-Trifluoromethylphenyl)benzene [T4-11, 753014-99-2, Ref. 17]

${ }^{1} \mathrm{H}$ NMR (400 MHz, $\left.\mathrm{CDCl}_{3}, \mathrm{TMS}\right): \delta 7.82(\mathrm{~s}, 3 \mathrm{H}), 7.80(\mathrm{~d}, 6 \mathrm{H}, J=8.4 \mathrm{~Hz}), 7.76(\mathrm{~d}, 6 \mathrm{H}, J=8.0 \mathrm{~Hz})$.

${ }^{13} \mathrm{C}$ NMR $\left(125 \mathrm{MHz}, \mathrm{CDCl}_{3}\right) \delta 144.2,141.8,130.3(\mathrm{q}, J=32.4 \mathrm{~Hz}), 127.9,126.4,126.2(\mathrm{q}, J=3.5 \mathrm{~Hz})$, $124.4\left(\mathrm{q}, J=270.4 \mathrm{~Hz}\right.$ ). Anal. Calcd for $\mathrm{C}_{27} \mathrm{H}_{15} \mathrm{~F}_{9}$ : C, 63.54; H, 2.96. Found: C, 63.59; H, 2.95.
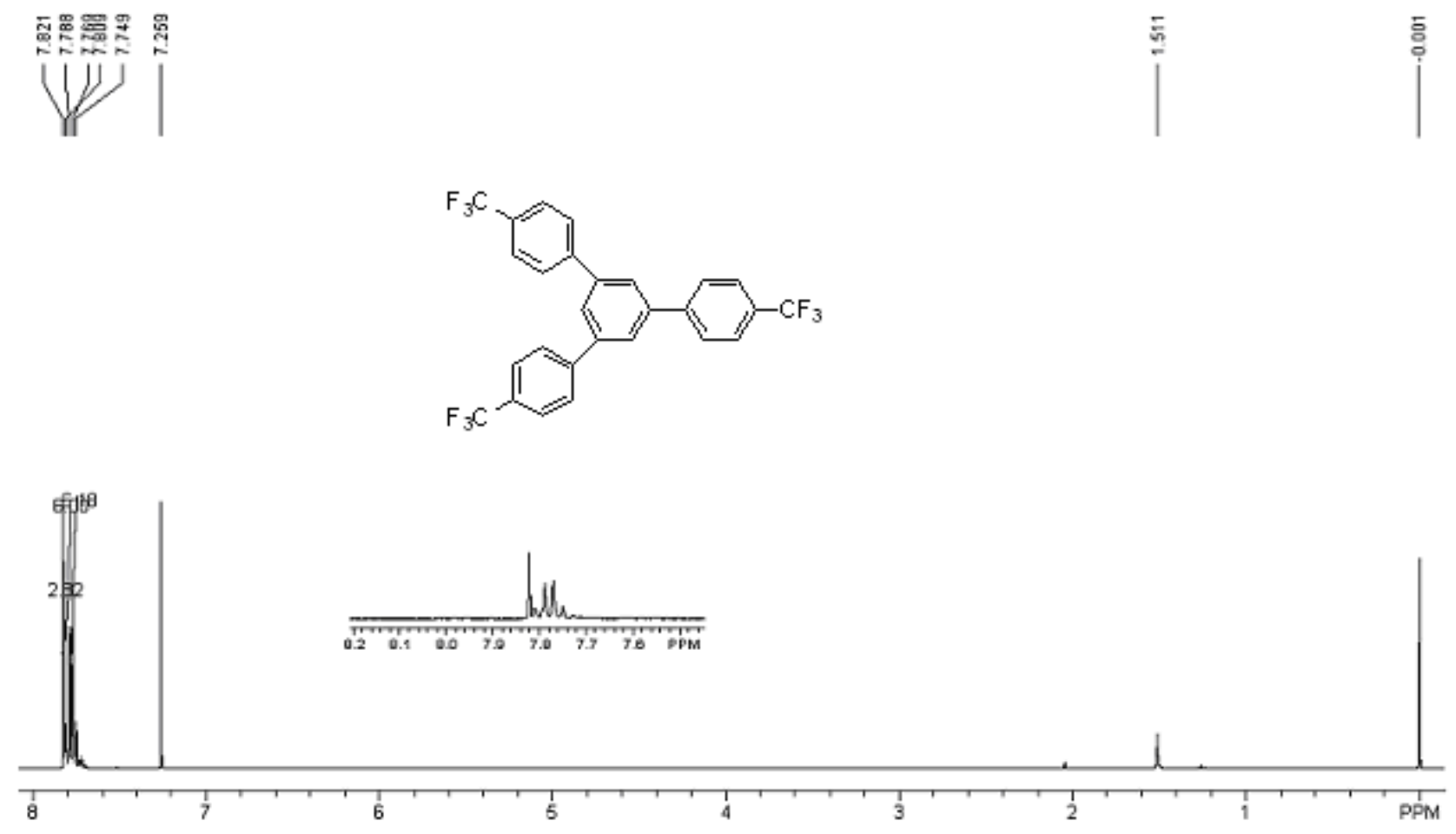

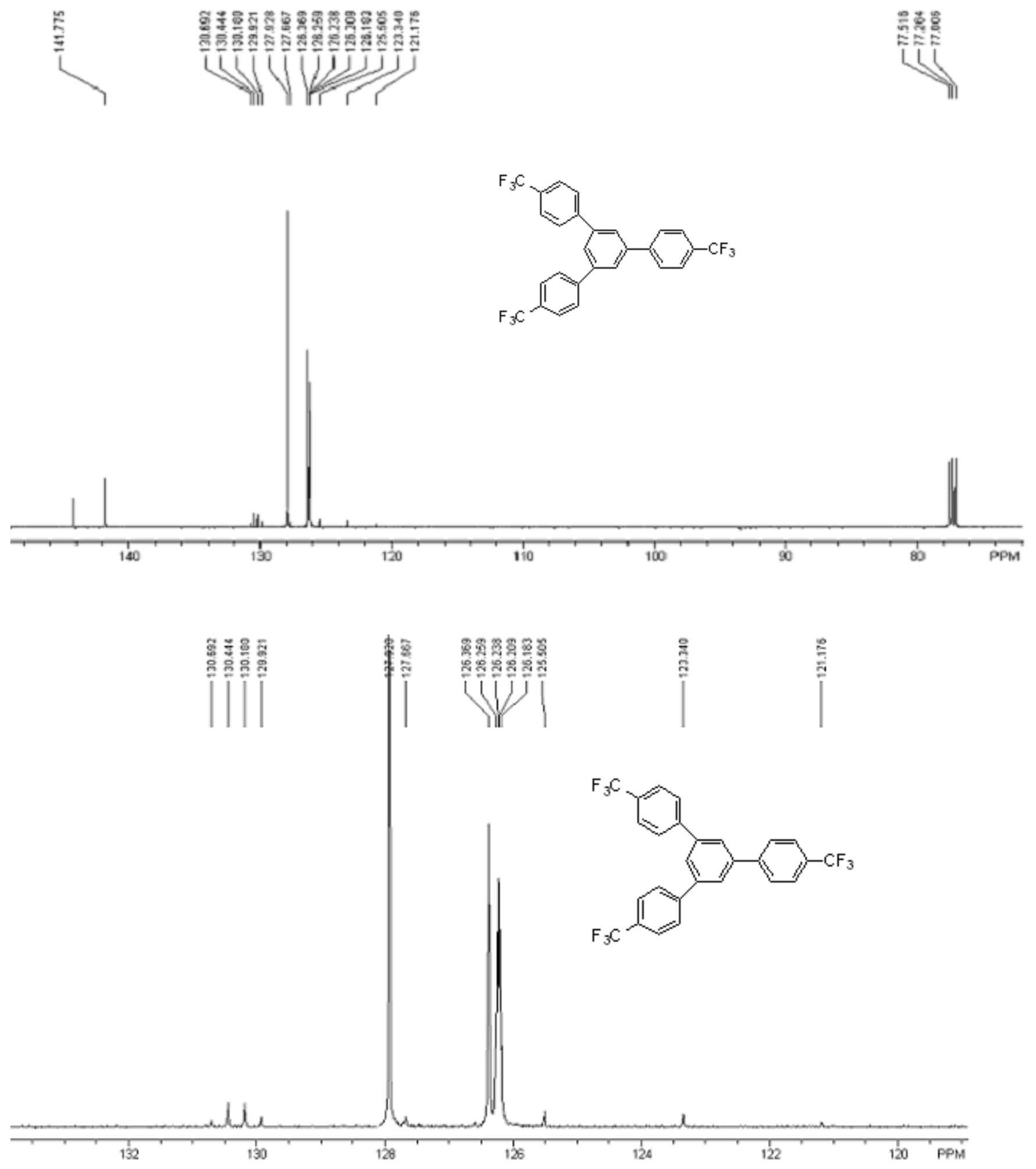


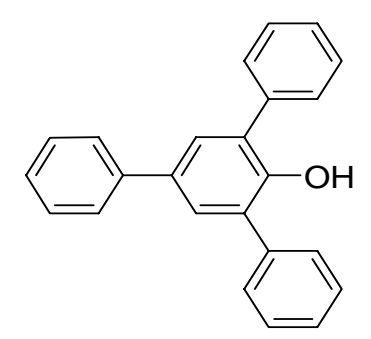

2, 4, 6-Triphenylphenol [T4-12, 3140-01-0, Ref. 18]

${ }^{1} \mathrm{H}$ NMR (500 MHz, $\mathrm{CDCl}_{3}$, TMS): $\delta 7.62(\mathrm{~m}, 6 \mathrm{H}), 7.53(\mathrm{~s}, 2 \mathrm{H}), 7.51(\mathrm{~m}, 4 \mathrm{H}), 7.42(\mathrm{~m}, 4 \mathrm{H}), 7.32$ (m, $1 \mathrm{H}) . \mathrm{MS}(\mathrm{ESI}): \mathrm{m} / \mathrm{z}=344.9\left([\mathrm{M}+\mathrm{Na}]^{+}\right)$.
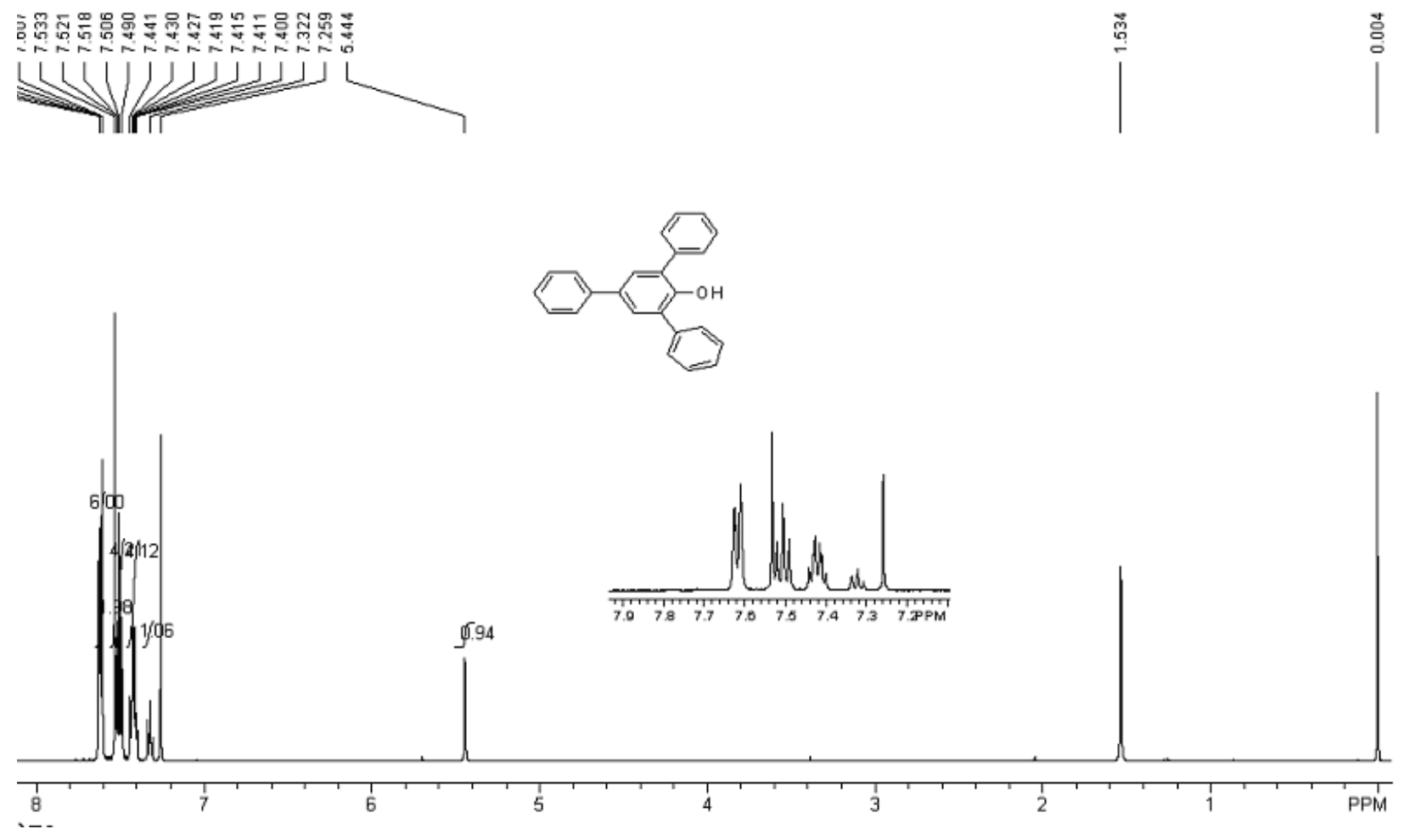
(28)

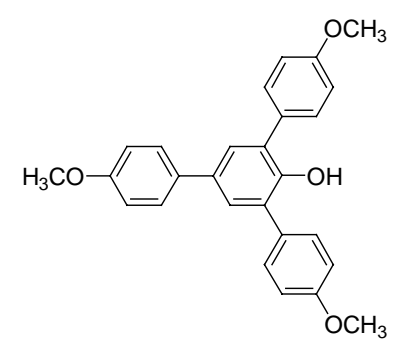

2, 4, 6-Tris(4-methoxyphenyl)phenol [T4-13, 6863-51-0, Ref. 19]

${ }^{1} \mathrm{H}$ NMR (400 MHz, $\mathrm{CDCl}_{3}$, TMS): $\delta 7.53(\mathrm{~d}, 6 \mathrm{H}, J=8.8 \mathrm{~Hz}$ ), $7.42(\mathrm{~s}, 2 \mathrm{H}), 7.25(\mathrm{~d}, 4 \mathrm{H}, J=8.8 \mathrm{~Hz}$ ), $6.95(\mathrm{~d}, 2 \mathrm{H}, \mathrm{J}=8.8 \mathrm{~Hz}), 5.37(\mathrm{~s}, 1 \mathrm{H}) . \mathrm{MS}(\mathrm{ESI}): \mathrm{m} / \mathrm{z}=413.0\left([\mathrm{M}+\mathrm{H}]^{+}\right), 434.9\left([\mathrm{M}+\mathrm{Na}]^{+}\right)$.
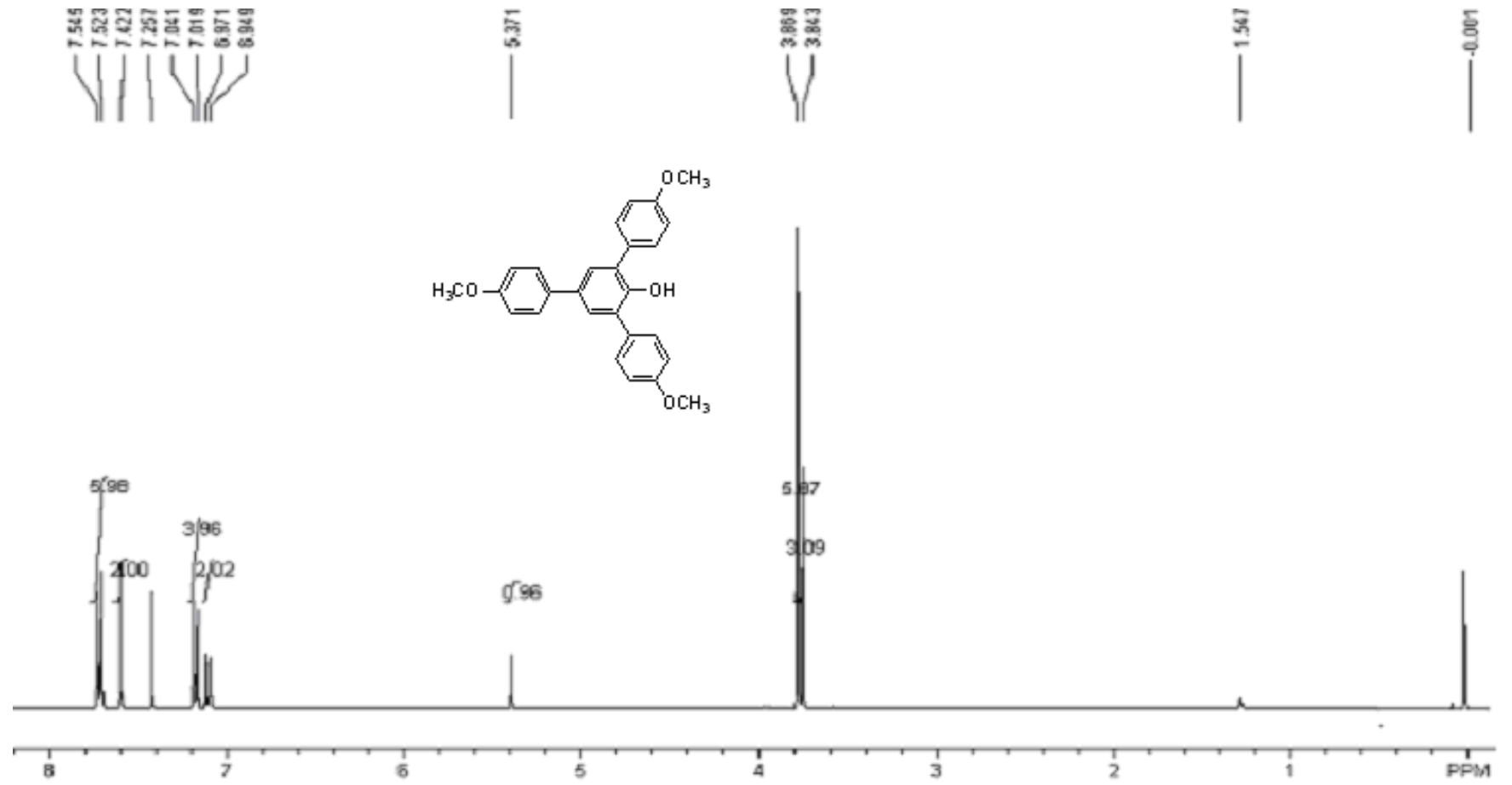
(29)

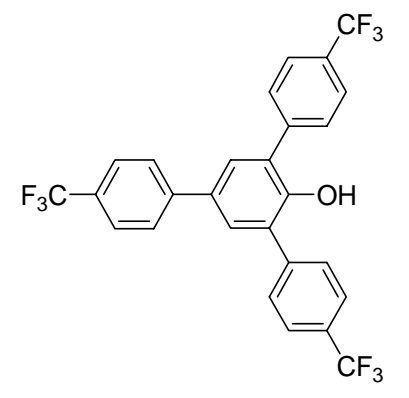

\section{2, 4, 6-Tris(4-Trifluoromethylphenyl)phenol [T4-14, New compound]}

${ }^{1} \mathrm{H}$ NMR (500 MHz, $\left.\mathrm{CDCl}_{3}, \mathrm{TMS}\right): \delta 7.79(\mathrm{~d}, 4 \mathrm{H}, J=8.5 \mathrm{~Hz}$ ), 7.73 (d, $4 \mathrm{H}, J=8.0 \mathrm{~Hz}), 7.70(\mathrm{~s}, 4 \mathrm{H})$, $7.55(\mathrm{~s}, 2 \mathrm{H}), 5.33(\mathrm{~s}, 1 \mathrm{H}) ;{ }^{13} \mathrm{C} \mathrm{NMR}\left(125 \mathrm{MHz}, \mathrm{CDCl}_{3}\right) \delta 149.7,143.7,140.8,133.3,130.6(\mathrm{q}, J=$ $32.3 \mathrm{~Hz}), 130.0,129.6(\mathrm{q}, J=32.3 \mathrm{~Hz}), 129.6,128.7,127.3,126.3(\mathrm{q}, J=3.4 \mathrm{~Hz}), 126.1(\mathrm{q}, J=3.6$ $\mathrm{Hz}), 124.5(\mathrm{q}, J=270.1 \mathrm{~Hz}), 124.3(\mathrm{q}, J=270.9 \mathrm{~Hz}) ; \mathrm{IR}(\mathrm{KBr}) v 3447,1617,1394,1328,1228,1164$, 1112, 1067, $837 \mathrm{~cm}^{-1}$; MS (ESI): m/z $525.0\left([\mathrm{M}-\mathrm{H}]^{+}\right.$); HRMS (ESI) calcd for $\mathrm{C}_{27} \mathrm{H}_{15} \mathrm{~F}_{9} \mathrm{O}, 525.0895$; found, 525.0893 .

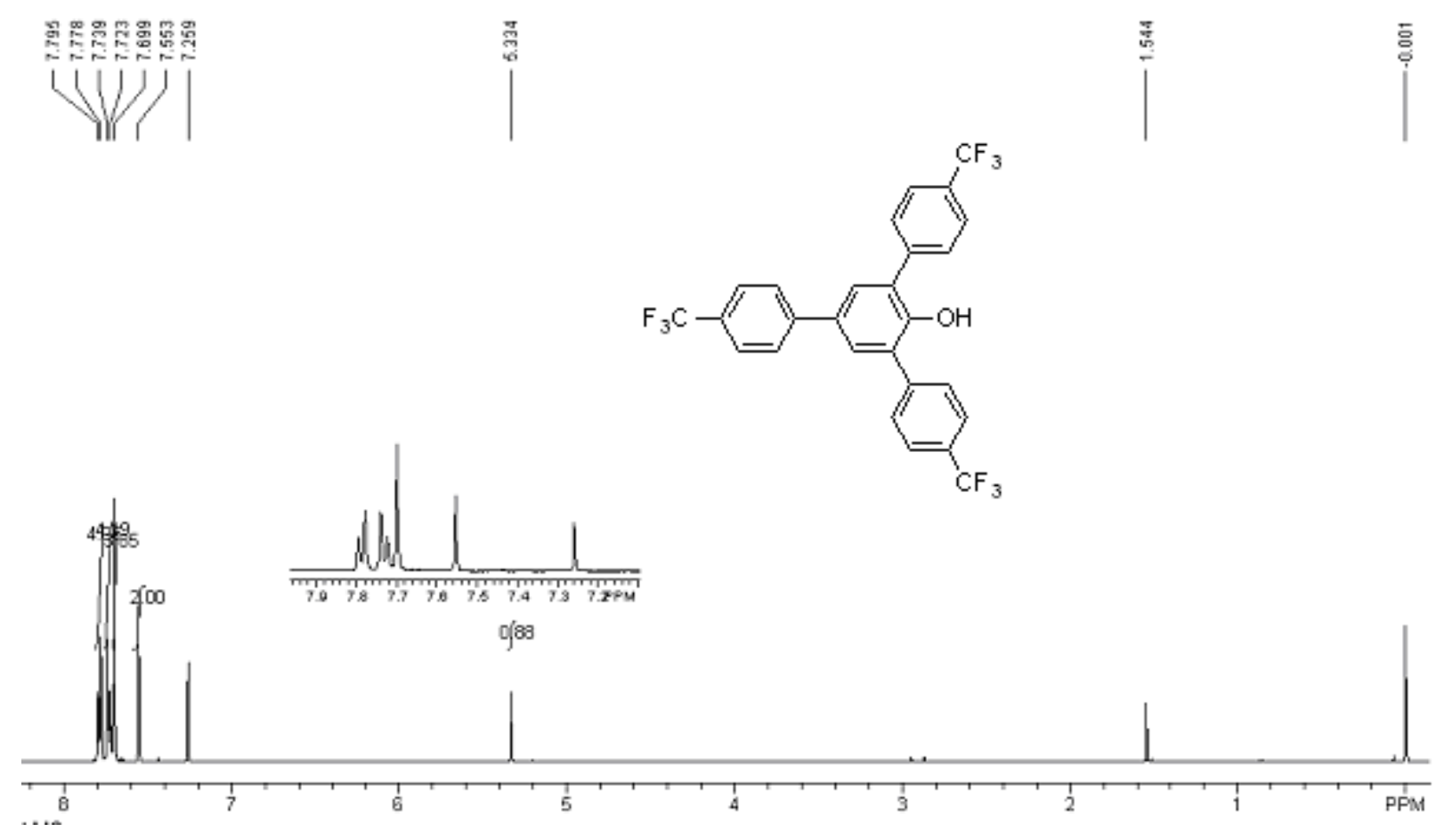



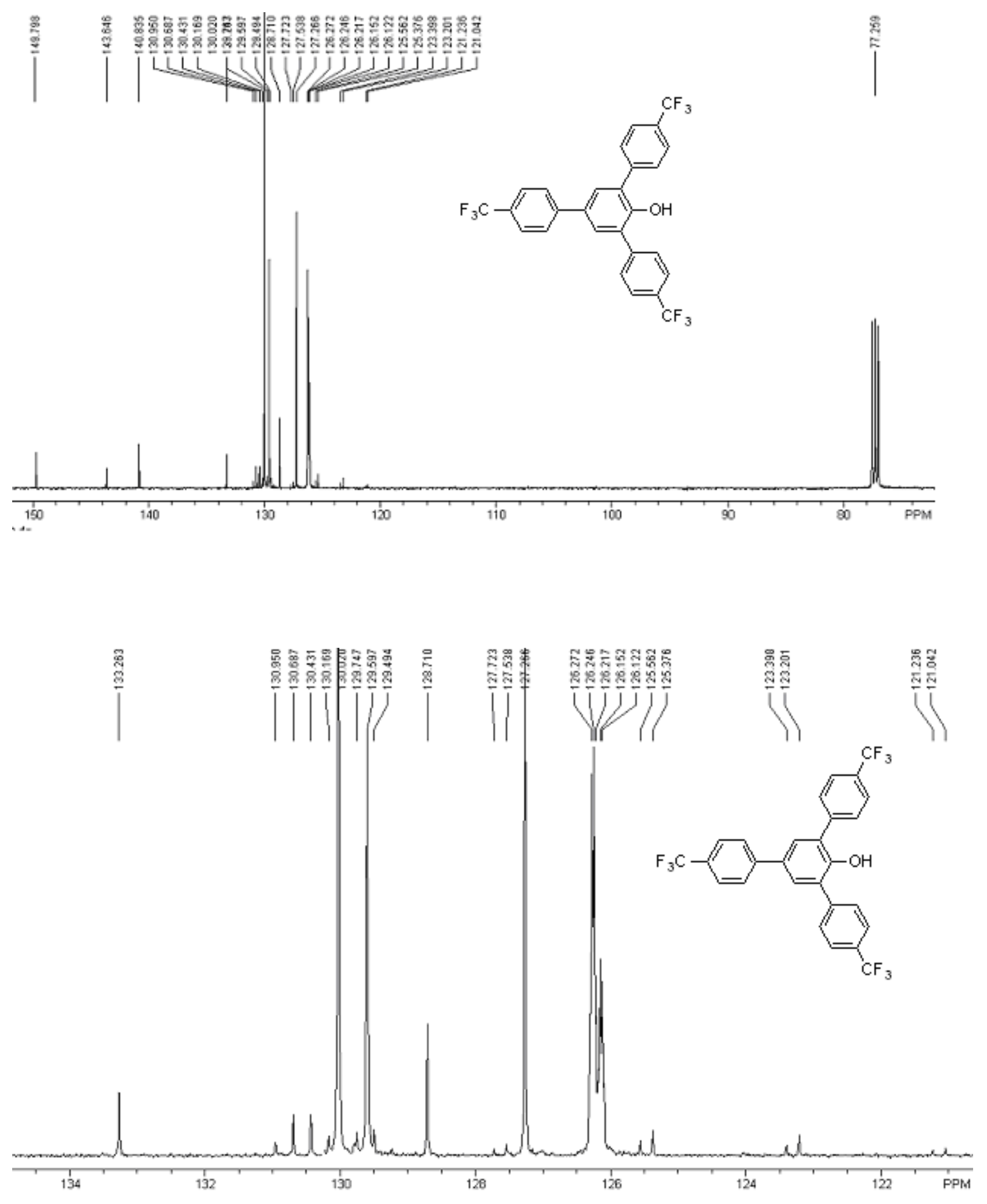


\section{Reference}

1. Tao, B.; Boykin, D.W. J.Org. Chem. 2004, 69, 4330-4335.

2.Yamada, Y. M. A.; Takeda, K.; Takahashi, H.; Ikegami, S. Org. Lett. 2002, 4, 3371-3374.

3. Leadbeater, N. E.; Marco, M. Org. Lett. 2002, 4, 2973-2976.

4. Sakurai, H.;Tsukuda, T.; Hirao, T. J. Org. Chem. 2002, 67, 2721-2722.

5. Navarro, O.; Kaur, H.; Mahjoor, P; Nolan, S. P. J.Org. Chem. 2003, 69, 3173-3180

6. Xiong, Z.; Wang, N.; Dai, M.; Li ,A.; Chen, J.; Yang, Z. Org. Lett. 2004, 6, 3337-3340.

7. Hennings, D. D.; Iwama, T.; Rawal. V. H. Org. Lett. 1999, 1, 1205-1208.

8. Wallow, T. I.; Novak, B. M. J.Org. Chem. 1994, 59, 5034-5037.

9. McNulty, J.; Capretta, A.; Wilson, J.; Dyck, J.; Adjabeng, G.; Robertson, A. Chem. Commun. 2002, 1986-1987.

10. Ikoma, Yoshiharu; Sugimori, Akira; Akiyama, Takeo, Jpn. Kokai Tokkyo Koho, JP 04282326 $19921007,1992$.

11. Tao, X.; Zhao, Y.; Shen, D. Synlett. 2004, 2, 359-361.

12. Sibille, S.; Ratovelomanana, V.; Nedelec, J. Y.; Perichon, J. Synlett. 1993, 6, 425-426.

13. Paul, S.; Clark, J. H. Green Chemistry, 2003, 5, 635-638.

14. Sinclair, D. J.; Sherburn, M. S. J.Org. Chem. 2005, 70, 3730-3733.

15. Zapka, W.; Brackmann, U. Applied Physics (Berlin), 1979, 20, 283-286.

16. Hu, Z. G.; Liu, J.; Li, G. A.; Dong, Z. B. Journal of Chemical Research, Synopses, 2003, 12, 778-779.

17. Fabbian, M.; Marsich, N.; Farnetti, E. Inorganica Chimica Acta 2004, 357, 2881-2888.

18. Basu, B.; Das, P.; Bhuiyan, M.; Mosharef ;H.; Jha, S. Tetrahedron Lett. 2003, 44, 3817-3820.

19. Dimroth, K.; Thomas, H. Chemische Berichte, 1969, 102, 3795-802. 